\title{
BEYOND EARTHQUAKES: The New Directions of ExPected Utility Theory*
}

\author{
FELIPE ZURITA** \\ Pontificia Universidad Católica de Chile
}

Over the past two decades, an enormous amount of work has been done to improve the Expected Utility model. Two areas have attracted major attention: the possibility of describing unforeseen contingencies and the need to accommodate the kind of behavior referred to in Ellsberg's paradox. This article critically assesses the Expected Utility model and its interpretations, as well as the basic principles underlying the referred extensions, with an emphasis on their applications.

JEL: D01, D81, D83

Keywords: Ambiguity, Unawareness, Unforeseen Contingencies, Expected Utility

*I am very grateful to Burkhard Schipper, who patiently clarified my doubts regarding his own work in a series of e-mails; to Federico Weinschelbaum for endless discussions; to Rodrigo Harrison and seminar participants at the Pontificia Universidad Católica de Chile for comments; to Edi Karni for an electronic exchange on Bayesian theory; and to Paolo Ghirardato for a long talk some years ago that brought this subject to my attention. Two anonymous referees made detailed observations and insightful comments, for which I am grateful. My special thanks to one of them, who catched several mistakes, made penetrating comments, and demanded a higher precision standard than the previous version aimed at, all of which substantially benefited the article. All remaining errors are naturally my responsibility.

**E-mail: fzurita@faceapuc.cl 
"But it does seem that the recent "incomplete market" studies may have left some of the most striking instances of incompleteness unstudied and in particular have given no indication how the Savage paradigm is to be deployed when we go beyond the weather and earthquakes".

Kenneth Arrow and Frank Hahn (1999)

\section{INTRODUCCIÓN}

Expected Utility Theory is a cornerstone of economic analysis. It rationalizes individual decision making in ignorance, that is, when the decision maker does not know the consequences of each possible action. As situations of this kind are widespread in economic life, it is not surprising that the theory has been so widely applied, to problems ranging from portfolio choice, gambling, insurance, investment, and education, to more intricate social phenomena such as coordination, delegation, or trading, to name just a few.

However, economists are finding a growing number of applications in which it seems incomplete and deficient, where observed behavior looks paradoxical through its lens. These paradoxes concern primarily more complex forms of ignorance. Consider, for instance, General Equilibrium Theory under uncertainty. Clearly, modern economies do not possess the type or the number of assets the model predicts, for it is extremely easy to find examples of untraded contingencies and, in that sense, asset markets are incomplete. The study of incomplete market economies, however, has typically taken the incompleteness as a starting point, without explaining its source (with a few exceptions, notably Allen and Gale, 1989). Arrow and Hahn (1999) close their perceptive discussion of the internal consistency of the model with our opening quotation. A basic message of theirs is that economists have in some sense abused Expected Utility Theory (henceforth EUT), putting it to work at tasks for which it was not designed, such as analyzing the sophisticated forms of uncertainty that characterize asset market transactions.

Over the past decade or so, much work has been done to produce a model that can handle these more complex forms of uncertainty, that is, that can go beyond earthquakes. This article reviews some of this work in order to asses its current state and promise. No attempt is made to thoroughly survey the literature. Instead, it discusses the main issues involved, with a particular emphasis on applications.

In particular, it focuses on two concepts that cannot be described in the language provided by Expected Utility Theory: unawareness and ambiguity.

The first refers to a situation in which the decision maker does not only ignore the consequence of each available act, but also does not even imagine all possible consequences, and hence may eventually find himself in an unforeseen situation. 
EUT's neglect of the higher level of ignorance posed by unawareness has important implications. For instance, a central result in Contract Theory asserts that two parties engaged in a long-term relationship will prefer to sign a complete contract over an incomplete one, meaning that all conflicts of interest that may arise in the relationship should be considered in the contract. This proposition is largely refuted by the evidence, as observed contracts are often vague and leave many contingencies undefined, which in turn often end up being renegotiated or legally disputed.

Speculation constitutes yet another example. The celebrated No Trade Theorem (Milgrom and Stokey, 1982) establishes that private information alone cannot be a motive for trade if there is common knowledge of rationality and all individuals have common priors. This latter assumption also involves their foreseeing a common set of contingencies; Heifetz, Meier and Schipper (2005) provide an example showing that this assumption is necessary for the No Trade Theorem. Thus, the development of an unforeseen contingencies theory may pave the way for a more intuitive and empirically relevant theory of speculation than we currently have.

In all these cases, extending EUT to accommodate the possibility of unawareness appears as a promising research area. This extension, however, has proved not to be simple, and the development of a tractable model is yet to come. Section 3 discusses the difficulties involved and promising new results as well.

The second strand of literature this article reviews is that of ambiguity, which in general terms investigates the behavioral consequences of non-additive representations of beliefs.

The EU model relies on probabilities to represent beliefs. The implications of this fact, however, depend on the meaning attached to the mathematical concept of probability. For instance, to an objectivist observer, who feels that a probability is part of the description of an object or process, like weight or mass, EUT is essentially a normative theory whose positive value depends on its predictive ability. This analyst, however, may wish to distinguish situations in which the probabilities are known to the decision maker from those where they are not. For him, EUT only applies to the former class of situations, while the study of the latter, when the decision maker may have an ambiguous belief, requires a different tool.

A subjectivist, on the other hand, understands probabilities as an expression of the (personal) doubts the decision maker entertains in a particular decision problem. He uses the EU model because if the decision maker's behavior obeys certain properties, then he knows that he can represent it as the result of the maximization of an EU function, regardless of the decision maker's level of knowledge or ignorance. He would rely on the EU model to predict behavior as long as it is empirically satisfactory. It turns out that in some classes of problems, it is not. As a consequence, the theory needs to be amended. The choice problem occurs under ambiguity if the observed behavior can be better predicted by a nonadditive belief. 
Section 4 discusses ambiguity. The review is not all-comprehensive, but focuses on identifying areas in which EUT's current use are problematic. Section 2, in turn, contains a general, schematic review of EUT.

I should stress that this article is intended for a general audience within the profession. While there are good surveys available on these topics (like those of Dekel et al., 1998b, on unawareness, and Ghirardato, 1993, on ambiguity) they tend to be too formal for many economists to fully grasp and apply. Instead, this article emphasizes interpretation, keeping the formal description to a minimum (although some is nonetheless necessary).

\section{The Expected Utility Model}

A choice problem occurs when a decision maker (henceforth DM) faces many mutually exclusive courses of action. Let $A$ represent the set that contains all those acts. If the DM knew the consequence of each, he could evaluate those consequences directly, using, for example, function $V(a)$. Choice Theory assumes such an evaluation is possible and deduces the $V$ function by looking at actual choices: the observed decision corresponds to the best evaluated among the available acts. Hence, if $a^{*}$ was chosen when $a$ was available, we infer that $V\left(a^{*}\right) \geq V(a)$ for all $a \in A$, or equivalently, that $a^{*} \in \arg _{\max } \operatorname{a\in A} V(a)$.

Uncertainty is a situation in which the DM does not know the consequence of each act. Although our usual image involves a DM who does not know what will happen in the future at the time when the decision is called for, the facts of which he is uncertain may or may not have already happened, or even not have a temporal dimension. For instance, the individual may not know if "it rained over Easter Island on July 23th, 621 A.D.," a fact of the past, or if "Pluto has two moons," a fact that lacks a temporal dimension -at least from the perspective of a regular human being. Conceptually, whether or not the DM knows something has already happened or will happen is immaterial; all that matters is that the DM does not know about it at the time of the decision, and therefore cannot predict the outcome of his acts.

We can nevertheless identify an important dimension involving time: the moment at which the DM is ignorant and must decide (call it ex ante), another when the decision has been made and he may find out about something but not everything (call it interim), and a final moment when the DM learns what the consequences of his decision were (call it ex post). These three instants are intimately related in Decision Theory.

The EU model assumes that ex ante the DM is capable of listing all possible scenarios or states of the world. A state of the world is a complete description of all relevant aspects of reality that explain a given consequence. Consider the following choice problem: 


\section{Example 1:}

A driver is deciding whether to take road 1 or road 2 to go home. Road 1 winds through a beautiful valley, but is often blocked by flocks of sheep. Road 2 is fast but gloomy, and is occasionally blocked by striking workers from the factories nearby. The DM knows about these characteristics, but does not know the truth value of the propositions $p \equiv$ "there is a flock blocking road 1 " and $q \equiv$ "a strike is under way, blocking road 2". The possible states of the world are hence defined by all the possible combinations of these two propositions and their negations (denoted by the sign " $\neg$ "). Let us denote by $\omega_{1}, \omega_{2}, \omega_{3}$, and $\omega_{4}$, respectively, the states defined by: $p \wedge q$ ("both roads are blocked"), $p \wedge \neg q$ ("road 1 is blocked and road 2 is not"), $\neg p \wedge q$ ("road 1 is free and road 2 is blocked"), and $\neg p \wedge \neg q$ ("both roads are free"), as shown in Table 1.

TABLE 1

STATES DEFINED BY PROPOSITIONS IN EXAMPLE 1

\begin{tabular}{ccc}
\hline & $p$ & $\neg p$ \\
\hline$q$ & $\omega_{1}$ & $\omega_{2}$ \\
$\neg q$ & $\omega_{3}$ & $\omega_{4}$ \\
\hline
\end{tabular}

Hence, a state of the world is specified by the truth value of all propositions that are relevant to the decision problem at hand, and as such it can be thought of as a completely specified compound proposition (as opposed to simple propositions, like $p$ or $q$ ). Knowing the state of the world is equivalent to knowing what the consequence of each act is or would have been, that is, a state of mind in which ignorance or uncertainty vanish. In our example, knowing that state $\omega_{3}$ obtains entails knowing that the choice of road 1 leads to a nice trip and reaching home on time, while road 2 to being home late after a rotten day.

If the set $\Omega$ contains the list of all possible states of the world, it is called the state space. We will always consider it a finite set. ${ }^{1}$ Observe that only one state is or will be "true" ex post, and all the rest will be declared to have looked plausible ex ante but proved "wrong" ex post. States are therefore mutually exclusive and jointly exhaustive.

An event $E$ is a subset of the state space, $\Omega$. $E$ is perhaps more naturally defined as the set of states at which a given proposition $p$ is true; for this reason we will denote indistinctively the event either by the set $E$ or by the proposition that generates it in brackets $[p]$. In Example 1, $p \equiv$ "there is a flock blocking road 1" corresponds to $[p]=\left\{\omega_{1}, \omega_{3}\right\}$. Its negation, $\neg p$, corresponds to the relative complement of $[p]$ with respect to $\Omega$, which we denote by $[\neg p] \equiv \Omega \backslash[p]=\left\{\omega_{2}, \omega_{4}\right\}$.

\footnotetext{
${ }^{1}$ An infinitely dimensional state space would make the discussion more difficult without substantially altering the conclusions on the issues we are interested on.
} 
Models that take as primitives the set of propositions are called syntactic, while those which take events as primitives are called semantic. ${ }^{2}$ Almost all economic models that deal with uncertainty or information are constructed around what Dekel et al. (1998a) label the standard state space model, the EU model being a prime example of it. The standard state space model is a semantic model that corresponds to a syntactic model in which in all states each given proposition is either true or false but not both (a condition they call real states), and where two propositions are considered equivalent if they induce the same event (the property of event sufficiency). These conditions turn out to have strong implications: it is impossible to describe unforeseen contingencies within a standard state space model, as Section 3 will explain.

Label $c(\omega, a)$ the consequence of act $a$ in state $\omega$ and $u(c(\omega, a))$ the ex post evaluation of consequence $c(\omega, a)$ (which will obtain under act $a$ if $\omega$ is the true state). The convention in utility theory is that "more of $u$ " means "better": the best evaluated possibility is assigned the highest value of $u$, and the worse the lowest. The real-valued function $u(c(\omega, a))$ is known as the Bernoulli or felicity function, and orders consequences according to their ex post evaluation or utility. A possible felicity function for Example 1 is the following:

TABLE 2

FELICITY OR BERNOULLI FUNCTION IN EXAMPLE 1

\begin{tabular}{lcccc}
\hline Act $\backslash$ State & $\omega_{1}$ & $\omega_{2}$ & $\omega_{3}$ & $\omega_{4}$ \\
\hline Take road 1 $\left(a_{l}\right)$ & 2 & 2 & 8 & 8 \\
Take road 2 $\left(a_{2}\right)$ & 1 & 5 & 1 & 5 \\
\hline
\end{tabular}

Observe that if only one state were considered possible, then the ex ante evaluation of the act may well be viewed as corresponding to the ex post evaluation of the consequence, that is $V(a)=u(c(\omega, a))$. This is in fact the usual convention in the timeless Choice Theory, where the ex ante and ex post instants are not distinguished. It is clear, however, that this cannot be so with uncertainty, as the evaluation of acts will vary with the states.

According to the Bernoulli function of Table 2, the DM would prefer to take road 1 if states $\omega_{1}, \omega_{3}$ or $\omega_{4}$ were true, and road 2 otherwise. But of course he does not know.

The EU model asks the DM in addition to hold numerically representable ex ante beliefs or degrees of trust in the truthfulness of each relevant proposition, and hence on each state. Moreover, these beliefs are assumed to be representable by a probability. Let $\pi(\omega)$ be the ex ante degree of confidence on $\omega$ being the true state.

The EU model holds that the DM behaves (i.e., chooses actions) as if maximizing a function such as this:

\footnotetext{
${ }^{2}$ Aumann (1999a, b) offers a comprehensive but rather technical comparison of these models.
} 


$$
V(a)=E[u(c(\omega, a))]=\sum_{\omega \in \Omega} \pi(\omega) u(c(\omega, a))
$$

that is, the function that evaluates acts (called generically utility function) corresponds to the expected value of the function that evaluates consequences (the Bernoulli or felicity function), hence the expected utility name.

Thus, if all states were considered equally likely, the driver of Example 1 would choose road 1 , since

$$
\begin{aligned}
& (2)\left(\frac{1}{4}\right)+(2)\left(\frac{1}{4}\right)+(8)\left(\frac{1}{4}\right)+(8)\left(\frac{1}{4}\right)=5>3=(1)\left(\frac{1}{4}\right) \\
& +(5)\left(\frac{1}{4}\right)+(1)\left(\frac{1}{4}\right)+(5)\left(\frac{1}{4}\right)
\end{aligned}
$$

The EU model is thus built on the following ideas: (i) The choice depends on an evaluation of the possible consequences (as opposed to principles, rules of thumb, etc., even though such ingredients may sometimes be considered part of the consequences); (ii) The list of possible consequences is complete, and defines a standard state space model; (iii) There is a probability function that describes the DM ex ante beliefs affecting choices, and (iv) The evaluation of consequences and probabilities has the particular, linear form of Equation (1).

As we have said, this combination produces a powerful tool. Nevertheless, the next two sections discuss separately some problems associated to (ii) and (iii)(iv), respectively.

\section{KNOWLEDGE AND UnAWARENESS}

This section holds on to the idea that and EU maximizer is a logicallyconsistent individual, but explores the possibility that his knowledge of the problem at hand is incomplete, in the sense that some possibilities may not cross his mind at the time the decision is called for. This does not mean that the likelihood of this event was deemed negligible, but rather the more extreme situation in which the DM does not foresee a possibility or is unaware of it.

The idea of unawareness complements the notion of ignorance quite naturally. If an individual ignores what will happen in the future, we say that he is uncertain. If he ignores some possibilities, we say that he is unaware of them. Note that we will say that anything the DM imagines as possible is indeed possible, while anything that is ex post observed to have happened but was not considered ex ante, was possible - an unforeseen contingency.

In introductory examples of EU theory this case does not arise: the DM is uncertain of the weather the following day and within the example it is obvious that rain/not rain exhaust all the possibilities. Or the DM is uncertain about whether an earthquake will occur before year's end. Or, at most, he is uncertain about the 
level of consumption he will achieve if he invests in a particular portfolio, and the possibilities are well covered by the positive real numbers. In all these examples, it would appear as logically impossible that a state from outside the specified state space materializes.

But it is not. All those descriptions reflect the way the DM sees the problem at hand with the information and resources available at the time of decision. However, his ex post evaluation of the act may as well be based on variables not considered ex ante. For instance, when purchasing an umbrella, a woman did not foresee that by choosing a fancy color she would be chased by confused bees. Her ex ante description of the problem did not include the bees that conformed her ex post account of the consequence of her decision.

Moreover, some decision problems are better described by an incomplete set of consequences. Consider the case of scientific research, where the outcome is not only uncertain, but often unimaginable. Similarly, consider the case of a writer who is planning to write a novel. She does not know what the finished novel will look like. So let's say she lists all possibilities: in her mind this must be all the books in the world, limited perhaps to her language, written, to be written, never to be written. This is logically plausible, as the writer knows all the letters and can certainly imagine all combinations of the characters, ${ }^{3}$ so in principle she could imagine all possible books and the process of writing would involve choosing from among those possibilities. The same would be true, of course, of the outcome of scientific research reports in the form of papers. Or more generally, of any ideas amendable of written communication, including of course, music.

This is clearly not possible, perhaps because of the brain's or cognition's physical restrictions. In the case of the writer, she probably cannot hold even one whole book in her mind at the same time. Observe, then, that the possibility of unforeseen contingencies is latent even in finitely dimensional state spaces (as in the book example, if we take the number of pages of every book to be bounded.)

In these examples, learning is not of the shrinking nature presented by Bayes' rule, where the DM uses the evidence to discard possibilities, but of an expanding nature: evidence may not help the individual discard states initially considered possible, which then do not materialize, but instead reveal other unforeseen events that actually happened or could happen in the future.

The reader may object to this as a criticism of EUT by pointing out that predictive, rather than descriptive accuracy is its purpose. Indeed, the fact that EUT does not describe the process of writing cannot be considered a major objection if it still predicts well. And yet, should not the model that predicts the best available contract between two parties anticipating future conflicting interest include a complete description of what each should do in every state of nature? As emphasized earlier, some EUT predictions do indeed refer to the description of contracts, laws, and market organization, issues in which the ex ante indescribability

${ }^{3}$ Reminiscent of the problem presented in Borges' short story "The Library of Babel" (in "The Garden of Forking Paths", Buenos Aires, Ed. Sur, 1941) 
of future contingencies is crucial. One must be aware of an event in order to have a belief about it and make a decision associated with it.

In Subsection 3.1 I discuss some difficulties involved in the extension of the EU model to incorporate unawareness and some possibly fruitful applications in Subsection 3.2.

\subsection{Modeling Unawareness}

I have said that $\Omega$ contains a list of all possibilities that ex ante the DM envisions for a given future date (or all the hypotheses he considers could be true). Only one state will obtain (or is true). The DM may not learn the truth under all circumstances, however. This may be represented by means of a possibility correspondence,

$$
\begin{aligned}
P: \Omega & \rightarrow 2^{\Omega} \backslash \varnothing \\
\omega & \rightarrow P(\omega)
\end{aligned}
$$

indicating at each state what states the DM considers to be possible in the interim.

In the particular case in which the individual always discovers the true state, we have $P(\omega)=\{\omega\}$ for all $\omega \in \Omega$. In general, however, it is possible that the DM is not completely sure about the occurrence of a state and entertains more than one possibility.

It is standard in economic analysis to impose on $P$ the following requirements:

$$
\begin{aligned}
& \text { P1. } \forall \omega \in \Omega, \quad \omega \in P(\omega) \\
& \text { P2. } \forall \omega^{\prime} \in P(\omega) \Rightarrow P\left(\omega^{\prime}\right)=P(\omega)
\end{aligned}
$$

P1 means that the DM always considers the true state to be possible. P2 states that if $\omega^{\prime}$ is considered to be possible when $\omega$ is the true state, then $\omega$ should be considered possible when $\omega^{\prime}$ is the true state as well. If $\omega^{\prime}$ and $\omega$ were considered possible at $\omega$ but only $\omega^{\prime}$ were deemed possible at $\omega^{\prime}$, then the DM could differentiate between those states just by asking herself if she considers each state to be possible or not; if she considers $\omega^{\prime}$ and $\omega$ to be possible, introspection should tell her she is not at $\omega^{\prime}$ for in that state she would consider $\omega$ to be impossible. Admitting the violation of P2 then would imply the acceptance of the possibility that the DM does not deduce everything her knowledge implies.

These assumptions imply that one can divide $\Omega$ into a collection of pairwise disjoint subsets of $\Omega$ whose union is $\Omega$ itself, that is, $P$ induces a partition of $\Omega{ }^{4}$ We will denote such partition by the letter $H$.

In Example 1, if the DM takes road 1, we can describe his epistemic status by two information functions: one to describe his ex ante status $\left(P_{0}\right)$, and the

${ }^{4}$ For a proof, see, for instance, Osborne and Rubinstein (1994), p. 68. 
other for his ex post status $\left(P_{l}\right)$. In turn, each induces a partition of $\Omega$, as Table 3 shows.

TABLE 3

POSSIBILITY CORRESPONDENCES AND THEIR ASSOCIATED

PARTITIONS OF $\Omega$

$$
\begin{array}{l|cc} 
& P_{0} & P_{l} \\
\hline \omega_{1} & \left\{\omega_{l}, \omega_{2}, \omega_{3}, \omega_{4}\right\} & \left\{\omega_{l}, \omega_{3}\right\} \\
\omega_{2} & \left\{\omega_{1}, \omega_{2}, \omega_{3}, \omega_{4}\right\} & \left\{\omega_{2}, \omega_{4}\right\} \\
\omega_{3} & \left\{\omega_{l}, \omega_{2}, \omega_{3}, \omega_{4}\right\} & \left\{\omega_{l}, \omega_{3}\right\} \\
\omega_{4} & \left\{\omega_{l}, \omega_{2}, \omega_{3}, \omega_{4}\right\} & \left\{\omega_{2}, \omega_{4}\right\} \\
H_{0}=\left\{\left\{\omega_{l}, \omega_{2}, \omega_{3}, \omega_{4}\right\}\right\} & \\
H_{1}=\left\{\left\{\omega_{l}, \omega_{3}\right\},\left\{\omega_{2}, \omega_{4}\right\}\right\}
\end{array}
$$

Ex ante the DM considers anything (in $\Omega$ ) to be possible; the associated partition is trivial. After taking road 1 , he learns wether there was a flock blocking road $1\left([p]=\left\{\omega_{1}, \omega_{3}\right\}\right)$ or not $\left([\neg p]=\left\{\omega_{2}, \omega_{4}\right\}\right)$. These two possible status are considered in $H_{l}$.

In our discussion, it is imperative to formally express ideas like "the DM knows $E$ ". With that purpose in mind, we define the knowledge operator from the possibility correspondence:

$$
K(E)=\{\omega \in \Omega \mid P(\omega) \subseteq E\}
$$

Equation 3 says that the DM knows that an event $E$ obtained if the proposition generating $E$ is true in every state he considers possible. Intuitively, since set inclusion is defined as implication of belonging (i.e., $\langle P(\omega) \subseteq E\rangle \Leftrightarrow\langle\omega \in P(\omega) \Rightarrow \omega \in E\rangle)$, knowledge of $P(\omega)$ involves knowledge of all events it implies. Similarly, $\neg K$ indicates that the individual does not know whether $E$ is true or not (which should not be confused with $K(\neg E)$ ), knowing that $\neg E$ obtained.)

It turns out that if the possibility correspondence satisfies P1 and P2, then the knowledge operator generated by Equation 3 satisfies the following properties: ${ }^{5}$

(K2) $\quad E \subseteq E^{\prime} \Rightarrow K(E) \subseteq K\left(E^{\prime}\right) \quad$ (monotonicity)

(K3) $\quad K(E) \cap K\left(E^{\prime}\right)=K\left(E \cap E^{\prime}\right) \quad$ (axiom of distribution)

\footnotetext{
${ }^{5}$ For a proof, see, for instance, Osborne and Rubinstein (1994), p. 70.
} 


$$
K(E) \subseteq E
$$

$$
K(E) \subseteq K(K(E))
$$$$
\neg K(E) \subseteq K(\neg K(E))
$$

(axiom of truth)

(positive introspection)

(negative introspection)

K1 asserts that in all states the DM knows the tautologies (indeed, all of them by event sufficiency). K2 says that if $E$ obtains then necessarily $E^{\prime}$ also obtains, then knowing $E$ implies knowing $E$ '. K3 says that the states for which he knows events $E$ and $E$ ' obtain are those in which he knows both events. K3 is actually implied by K2. K4 says that if the DM knows an event, then that event indeed obtains; put another way, the DM cannot know a proposition if this is false. $\mathrm{K} 5$ requires the DM to know his knowledge: if he knows $E$, he must know that he knows it. K6 further requires that if he does not know something, he must know that he does not know it.

An intuitive definition of unawareness would declare an individual to be unaware of $E$ when the individual not only does not know $E$, but also does not know that he does not know it:

$$
U(E) \equiv \neg K(E) \cap \neg K(\neg K(E))
$$

Yet the above described knowledge operator precludes this possibility. Combining K4 with K6, we obtain $\neg K(E)=K(\neg K(E))$, so that $\neg K(E) \cap \neg K(\neg K(E))=\varnothing$ : in no state can the DM be unaware of some event.

This observation lead many authors to consider relaxing K6 (and hence abandoning partitional possibility correspondences) in order to be able to model unawareness. Consider the following example (taken from Dekel et al. 1998a, referred to from now on as DLR, who in turn adapted it from Geanakoplos, 1989).

\section{Example 2}

"While Watson never reported it, Sherlock Holmes once noted an even more curious incident, that of a dog that barked and the cat that howled in the night. When Watson objected that the dog did not bark and the cat did not howl, Holmes replied 'that is the curious incident to which I refer.' Holmes knew that this meant that no one, neither man nor dog, had intruded on the premises the previous night. For had a man intruded, the dog would have barked. Had a dog intruded, the cat would have howled. Hence, the lack of either of these two signals means that there could not have been a human intruder or canine intruder."

If $h=$ "there is a human intruder" and $c=$ "there is a canine intruder", then the equivalent to Table 1 for Example 1 is Table 4 below. Observe that two intruders are not possible in this example, so that the state space is $\Omega=\left\{\omega_{1}, \omega_{2}, \omega_{3}\right\}$. Recall also that event sufficiency means in this example that the propositions "there is a human intruder" and "the dog barks" are equivalent, as their truth values are the same in every state; similar comment applies to "there is a canine intruder" and "the cat howls". 
TABLE 4

STATES DEFINED BY PROPOSITIONS IN EXAMPLE 2

\begin{tabular}{c|cc} 
& $h$ & $\neg h$ \\
\hline$c$ & - & $\omega_{2}$ \\
$\neg c$ & $\omega_{1}$ & $\omega_{3}$
\end{tabular}

Following DLR, Watson's possibility correspondence would be as in Table 5:

TABLE 5

WATSON'S POSSIBILITY CORRESPONDENCE

\begin{tabular}{c|c}
$\omega$ & $P_{W}(\omega)$ \\
\hline$\omega_{l}$ & $\left\{\omega_{l}\right\}$ \\
$\omega_{2}$ & $\left\{\omega_{2}\right\}$ \\
$\omega_{3}$ & $\left\{\omega_{l}, \omega_{2}, \omega_{3}\right\}$
\end{tabular}

meaning that when there is a human intruder or a canine intruder, he is aware of its presence, but when there is no intruder he considers possible all three states, because he fails to realize that had a man or a dog intruded, he would know it, and therefore, as he does not know it, the state must be $\omega_{3}$. In contrast, the logicallysophisticated Holmes has the possibility correspondence $P_{H}$ :

TABLE 6

HOLMES' POSSIBILITY CORRESPONDENCE

\begin{tabular}{c|c}
$\omega$ & $P_{W}(\omega)$ \\
\hline$\omega_{l}$ & $\left\{\omega_{l}\right\}$ \\
$\omega_{2}$ & $\left\{\omega_{2}\right\}$ \\
$\omega_{3}$ & $\left\{\omega_{3}\right\}$
\end{tabular}

To be sure, Watson's knowledge system fails to satisfy K6, negative introspection, which can be appreciated by comparing the last two columns of Table 7. For instance, in state $\omega_{3}$ he knows that he does not know $\left\{\omega_{l}\right\}$, but he does not know this fact: $K\left(\neg K\left(\left\{\omega_{3}\right\}\right)\right) \supseteq \neg K\left(\left\{\omega_{3}\right\}\right)$. 
TABLE 7

WATSON'S KNOWLEDGE STRUCTURE

\begin{tabular}{c|ccccc}
$E$ & $P(\omega)$ & $K(E)$ & $K(K(E))$ & $\neg K(E)$ & $K(\neg K(E))$ \\
\hline$\varnothing$ & $\varnothing$ & $\varnothing$ & $\varnothing$ & $\Omega$ & $\Omega$ \\
$\left\{\omega_{1}\right\}$ & $\left\{\omega_{1}\right\}$ & $\left\{\omega_{1}\right\}$ & $\left\{\omega_{1}\right\}$ & $\left\{\omega_{2}, \omega_{3}\right\}$ & $\left\{\omega_{2}\right\}$ \\
$\left\{\omega_{2}\right\}$ & $\left\{\omega_{2}\right\}$ & $\left\{\omega_{2}\right\}$ & $\left\{\omega_{2}\right\}$ & $\left\{\omega_{1}, \omega_{3}\right\}$ & $\left\{\omega_{1}\right\}$ \\
$\left\{\omega_{3}\right\}$ & $\left\{\omega_{1}, \omega_{2}, \omega_{3}\right\}$ & $\varnothing$ & $\varnothing$ & $\Omega$ & $\Omega$ \\
$\left\{\omega_{1}, \omega_{2}\right\}$ & - & $\left\{\omega_{1}, \omega_{2}\right\}$ & $\left\{\omega_{1}, \omega_{2}\right\}$ & $\{c\}$ & $\varnothing$ \\
$\left\{\omega_{1}, c\right\}$ & - & $\left\{\omega_{1}\right\}$ & $\left\{\omega_{1}\right\}$ & $\left\{\omega_{2}, \omega_{3}\right\}$ & $\left\{\omega_{2}\right\}$ \\
$\left\{\omega_{2}, \omega_{3}\right\}$ & - & $\left\{\omega_{2}\right\}$ & $\left\{\omega_{2}\right\}$ & $\left\{\omega_{1}, \omega_{3}\right\}$ & $\left\{\omega_{1}\right\}$ \\
$\Omega$ & - & $\Omega$ & $\Omega$ & $\varnothing$ & $\varnothing$
\end{tabular}

We can check in Table 8 (continued from Table 7) that at $\omega_{3}$ Watson seems to be unaware of $\left\{\omega_{l}\right\}$ : not only at that state does he not know of the event $\left\{\omega_{l}\right\}$ ( $\left.\omega_{3} \notin K\left(\left\{\omega_{l}\right\}\right)=\left\{\omega_{l}\right\}\right)$, but also he does not know that he does not know it $\left(\omega_{3} \notin K\left(\neg K\left(\left\{\omega_{l}\right\}\right)\right)=\left\{\omega_{2}\right\}\right)$, that is, he does not know of his lack of knowledge.

TABLE 8

WATSON'S UNAWARENESS STRUCTURE

\begin{tabular}{c|ccc}
$E$ & $\neg K(E)$ & $\neg K(\neg K(E))$ & $U(E)$ \\
\hline$\varnothing$ & $\Omega$ & $\varnothing$ & $\varnothing$ \\
$\left\{\omega_{1}\right\}$ & $\left\{\omega_{2}, \omega_{3}\right\}$ & $\left\{\omega_{1}, \omega_{3}\right\}$ & $\left\{\omega_{3}\right\}$ \\
$\left\{\omega_{2}\right\}$ & $\left\{\omega_{1}, \omega_{3}\right\}$ & $\left\{\omega_{2}, \omega_{3}\right\}$ & $\left\{\omega_{3}\right\}$ \\
$\left\{\omega_{3}\right\}$ & $\Omega$ & $\varnothing$ & $\varnothing$ \\
$\left\{\omega_{1}, \omega_{2}\right\}$ & $\{c\}$ & $\Omega$ & $\left\{\omega_{3}\right\}$ \\
$\left\{\omega_{1}, \omega_{3}\right\}$ & $\left\{\omega_{2}, \omega_{3}\right\}$ & $\left\{\omega_{1}, \omega_{3}\right\}$ & $\left\{\omega_{3}\right\}$ \\
$\left\{\omega_{2}, \omega_{3}\right\}$ & $\left\{\omega_{1}, \omega_{3}\right\}$ & $\left\{\omega_{2}, \omega_{3}\right\}$ & $\left\{\omega_{3}\right\}$ \\
$\Omega$ & $\varnothing$ & $\Omega$ & $\varnothing$
\end{tabular}

DLR show, however, that this example has an awkward feature: while $U\left(\left\{\omega_{l}\right\}\right)=\left\{\omega_{3}\right\}$, that is, at $\omega_{3}$ Watson is unaware of $\left\{\omega_{l}\right\}$, at the same time $U\left(U\left(\left\{\omega_{1}\right\}\right)\right)=U\left(\left\{\omega_{3}\right\}\right)=\varnothing$, that is, Watson is aware (i.e. not unaware) of the fact that he is unaware of $\left\{\omega_{l}\right\}$. While he could be aware of or even know he is unaware of something, it does not make sense to say that he is aware of precisely what facts he is unaware of.

Moreover, they show that this feature is not specific to this example, but common to all standard state-space models. This is a devastating result that militates against the idea of amending the model, as suggested by Geanakoplos 
(1989). There are other ways, nonetheless. In particular, they blame necessitation (K1) and monotonicity (K2) instead of negative introspection (K6) for this. Below we will review two propositions for incorporating unawareness into the model.

Rather than proposing a new model, Ely (1998) presents a reconciling interpretation. He argues that the breakdown of negative introspection is not intended to reflect logical inconsistencies in the reasoning of the DM, but rather his failure to capture "the whole picture" (which the modeler sees), i.e., his lack of understanding of the way his perception works, which also happens to be the source of his unawareness.

Ely conjectures that this can be captured by a model based on a collection of partitions of $\Omega,\left\{H_{\omega}\right\}$, one for each state, representing the DM's understanding of the situation at each state. The modeler knows that at state $\omega$ the thinking of the DM will be characterized by $H_{\omega}(\omega)$, although at that state, the DM thinks that at state $\omega^{\prime}$ he would have thought according to $H_{\omega}\left(\omega^{\prime}\right)$. Since at each $\omega$ his possibility correspondence is a partition, the DM's model is internally consistent in the sense provided by conditions K1-K6. Thus, Watson's case could be modeled in the following fashion:

TABLE 9

WATSON'S POSSIBILITY CORRESPONDENCES IN ELY'S VIEW

\begin{tabular}{c|ccc}
$\omega$ & $P_{\omega_{l}}^{W}(\omega)$ & $P_{\omega_{2}}^{W}(\omega)$ & $P_{\omega_{3}}^{W}(\omega)$ \\
\hline$\omega_{1}$ & $\left\{\omega_{1}\right\}$ & $\left\{\omega_{1}, \omega_{3}\right\}$ & $\left\{\omega_{1}, \omega_{2}, \omega_{3}\right\}$ \\
$\omega_{2}$ & $\left\{\omega_{2}, \omega_{3}\right\}$ & $\left\{\omega_{2}\right\}$ & $\left\{\omega_{1}, \omega_{2}, \omega_{3}\right\}$ \\
$\omega_{3}$ & $\left\{\omega_{2}, \omega_{3}\right\}$ & $\left\{\omega_{1}, \omega_{3}\right\}$ & $\left\{\omega_{1}, \omega_{2}, \omega_{3}\right\}$
\end{tabular}

Observe that while the modeler "sees" the main diagonal, reconstructing Table 5 and recognizing Watson's violation of negative introspection, Watson does not know what he would have thought in other states, and is unaware of some possibilities. At state $\omega_{3}$ he thinks everything is possible and holds the logically consistent (but incorrect) belief that he would have thought the same had state $\omega_{l}$ being true.

Under this approach, the criticism that DLR raises over the unawareness operator of Equation 4 is no longer valid: when applied to the modeler's understanding of the situation, it is fine for him to say that at $\omega_{3}$ Watson is unaware of $\left\{\omega_{l}\right\}$, and the modeler is aware of this fact, but not Watson. Observe that at $\omega_{3}, K_{\omega_{3}}\left(\left\{\omega_{1}\right\}\right)=\varnothing$, and also $K_{\omega_{3}}\left(\left\{\omega_{1}, \omega_{3}\right\}\right)=\varnothing$, so in no state does he know that there was a human intruder or its negation.

Heifetz, Meier and Schipper (2005, henceforth HMS), on the other hand, propose an extended model, which not only allows for a form of unawareness immune to the DLR critique, but also has the advantage of being capable of incorporating multiple individuals, and speaking of "interactive unawareness." This is certainly a must for the theory to be useful for analyzing social problems. 
Although I will focus on individual decision making here, the applications discussed in the following subsection will give a flavor of such interaction.

HMS allow for personal or subjective state spaces. An external observer can imagine a whole set of such spaces, and see that the DM resides in one of them. In each of those spaces there is a limited set of concepts (propositions), so that their expressive powers differ. Observe that one can (partially) order those spaces by their expressive powers. The lowest level space is completely empty: a DM living in such space has essentially no conscience whatsoever. The highest level space is akin to the standard state space model's $\Omega$ : anything that is relevant can be thought about in it. Whoever lives in it is omniscient in that sense. In between those extremes there are many other spaces, where some concepts are present and others absent; the DM that resides in a particular space is unaware of any concept that is absent in it.

In Example 2, where the two relevant propositions are $h=$ "there is a human intruder" and $c=$ "there is a canine intruder", there are four state spaces: $\Omega_{c h}, \Omega_{h}, \Omega_{c}$ and $\Omega_{\varnothing}$. These are illustrated in Diagram 1, where each box represents one such space. Inside the box, the first line indicates what (basic) propositions can be said or thought within the corresponding space. The second line describes the state space those propositions give rise to. The lines connecting the boxes show which spaces have more expressive power, in ascending order. The set of spaces is only partially ordered, because there are spaces that cannot be compared in terms of expressive power; this is the case with spaces $\Omega_{h}$ and $\Omega_{c}$. Let us now denote by $\Omega$ the union of all spaces. This set contains all states that an omniscient external observer could think of.

DIAGRAM 1

THE ORDERED SET OF STATE SPACES IN HMS

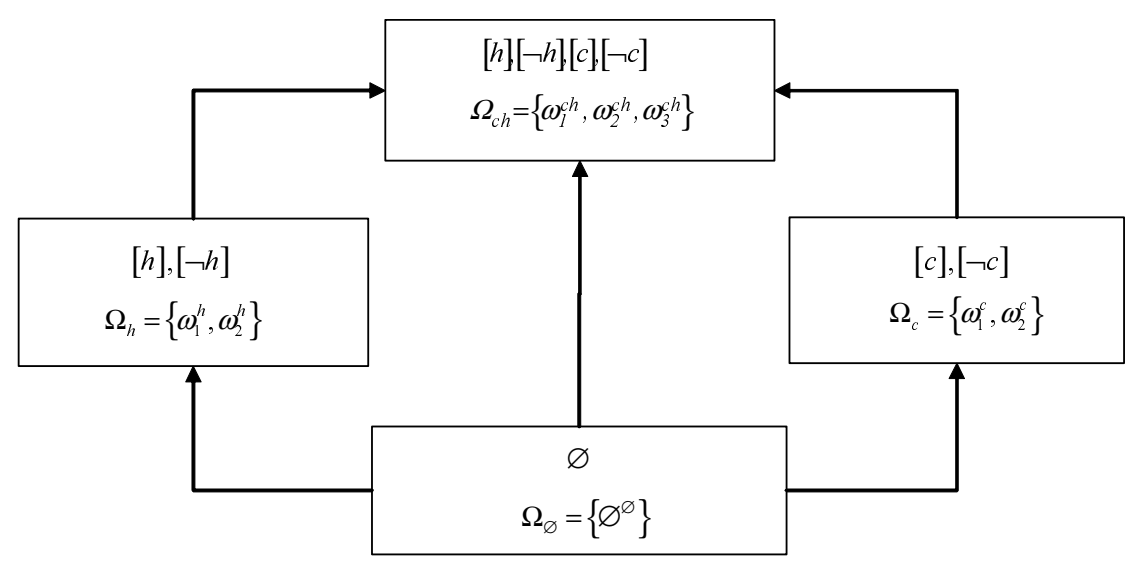

A DM that lives in $\Omega_{h}$ is aware of $h$ (and hence of its negation). Moreover if her information partition of $\Omega_{h}$ distinguishes $h$ from $\neg h$, when $h$ is true she 
knows that state $\varpi_{l}^{h}$ obtained. At the same time, for the inhabitants of $\Omega_{c}$ and $\Omega_{\varnothing}$ nothing happened. From the viewpoint of the external observer, the event $[h]=\left\{\varpi_{l}^{h}, \varpi_{l}^{c h}\right\}$ obtained. Observe that now states in one space correspond to events in higher spaces.

Observe also that from the scientist's point of view, the description of the event involves all states or events from each of the spaces where that event is describable. There is an important feature of this model with regard to the algebra of events: since the negation of event $[h]=\left\{\varpi_{l}^{h}, \varpi_{l}^{c h}\right\}$ is $[\neg h]=\left\{\varpi_{2}^{h}, \varpi_{2}^{c h}, \varpi_{3}^{c h}\right\},[h] \cup[\neg h] \neq \Omega$, that is, there are states that belong to neither event. Hence, this is not a standard state space model (because it fails to have real states). Those states belong precisely to spaces where the proposition is not expressively, that is, where individuals are unaware of it. Indeed, a state is a complete description of the world from the DM's point of view, and hence it is as incomplete as the DM's understanding of the situation. That incompleteness is understood by the external observer; hence, the state describes the truth value of all propositions regarding "facts of nature" the DM is aware of, and the propositions she is unaware of.

In the standard state space model, $\Omega$ represented all tautologies, i.e., propositions of the form $p \vee \neg p$ and $\varnothing$ all contradictions, i.e. proposition of the form $p \wedge \neg p$. In contrast, since in HMS's model not all propositions are expressively in each space, a proposition like $p \vee \neg p$ could either be tautological or completely meaningless. Then, just as in general we have $\Omega_{\pi} \neq \Omega_{\theta}$, we also have $\varnothing_{\pi} \neq \varnothing_{\theta}$. In particular, the state $\varnothing \varnothing$ is both a contradiction and a tautology in the space $\Omega_{\varnothing}$.

Within each space, the possibility correspondences can be partitional. From the DM's point of view, her knowledge satisfies K1-K6. It is the external observer who notices the failure of $\mathrm{K} 6$.

Going back to Example 2, we note that each DM may reside in different spaces depending on the circumstances. Holmes naturally lives in the highest space $\Omega_{c h}$-that is why we admire him so much after all. Watson, being a more straightforward person, lives in different spaces depending on the external stimuli he perceives. When proposition $h$ is true, he notices it, and consequently is also able to think of the counterfactual state in which there was no human intruder-but this is so only after noticing there was in fact a human intruder. Moreover, in that case the possibility of a canine intruder doesn't cross Watson's mind. Hence, when $h$ is true Watson lives in $\Omega_{h}$. Likewise, when proposition $c$ is true Watson lives in $\Omega_{c}$. When both of them are false, i.e. he sees nothing, neither possibility is present in his thought; this is to say, he lives in $\Omega_{\varnothing}$. This is summarized by his possibility correspondences in Table 10. 
TABLE 10

WATSON'S POSSIBILITY CORRESPONDENCES FROM WATSON'S VIEWPOINT

\begin{tabular}{c|ccc} 
Proposition & $P_{[h \wedge \neg c]}^{W}(\omega)$ & $P_{[\neg h \wedge c]}^{W}(\omega)$ & $P_{[\neg h \wedge \neg c]}^{W}(\omega)$ \\
\hline$[h \wedge \neg c]$ & $\left\{\omega_{1}^{h}\right\}$ & $\left\{\omega_{2}^{c}\right\}$ & $\{\varnothing \varnothing\}$ \\
{$[\neg h \wedge c]$} & $\left\{\omega_{2}^{h}\right\}$ & $\left\{\omega_{1}^{c}\right\}$ & $\{\varnothing \varnothing\}$ \\
{$[\neg h \wedge \neg c]$} & $\left\{\omega_{2}^{h}\right\}$ & $\left\{\omega_{2}^{c}\right\}$ & $\{\varnothing \varnothing\}$
\end{tabular}

Observe the similarity to Ely's proposition (Table 9). The intuitive idea is in fact the same; HMS's contribution is to work out a formal model where that intuitive idea can be properly expressed.

\subsection{Applications of Unawareness}

This subsection discusses two examples of problems where the explicit consideration of unawareness may boost our understanding.

\subsubsection{The no-trade theorem}

Information transmission in financial markets has been studied theoretically ever since the development of information economics. While some authors sought to describe in a model a situation where investors would trade based on disparate information (Lintner 1969, Hirshleifer 1977) generating divergent opinions over capital gains, or speculation, economists promptly abandoned this idea on the grounds that rationality would preclude it as an equilibrium phenomenon. Thus, Radner (1979) showed that if investors knew the connection between asset prices and states, then generically perfectly competitive equilibrium prices would be perfectly revealing. This is to say that disparately informed traders would deduce (almost) ${ }^{6}$ all information possessed by their peers from the (perfectly competitive) equilibrium prices of assets.

By the same token, Milgrom and Stokey (1982) showed that if it is commonly known that there are no reasons to trade apart from private information, and if differences of opinion can only arise from differences in information, then in any Nash equilibrium there is no trade and information is (almost) completely revealed. The result holds even in more general trading environments than perfectly competitive markets.

\footnotetext{
${ }^{6}$ The 'almost' qualification arises from the fact that two states may result in the same equilibrium price. In this case, however, the differences in information between traders are of no use to them.
} 
To be concrete, consider a situation involving two propositions, $p$ and $q$, and let us analyze it under the standard state space model. Individual A owns one unit of an asset, and observes privately the truth value of $p$ (i.e., observes whether an event $E$ obtains or not). Individual B is a would-be buyer, who privately observes the truth value of $q$ (i.e., observes whether an event $F$ obtains or not). The asset will have in the next period a liquidation value that depends on both events as in Table 11.

TABLE 11

STATE-CONTINGENT LIQUIDATION VALUES FOR THE TRADING EXAMPLE

\begin{tabular}{lcccc}
\hline & \multicolumn{2}{c}{$q$} & $\neg q$ \\
\hline$p$ & $100-20+20$ & $\left(\omega_{1}\right)$ & $100-20$ & $\left(\omega_{2}\right)$ \\
$\neg p$ & $100+20$ & $\left(\omega_{3}\right)$ & 100 & $\left(\omega_{4}\right)$ \\
\hline
\end{tabular}

Hence, A has an information partition $H_{A}=\left\{\left\{\omega_{1}, \omega_{2}\right\},\left\{\omega_{3}, \omega_{4}\right\}\right\}$ while B has $H_{B}=\left\{\left\{\omega_{1}, \omega_{3}\right\},\left\{\omega_{2}, \omega_{4}\right\}\right\}$. Learning $[p]=\left\{\omega_{1}, \omega_{2}\right\}$ obtained is bad news for A, and an incentive to sell. Similarly, learning $[q]=\left\{\omega_{l}, \omega_{3}\right\}$ obtained is good news for B, and an incentive to buy. However, if they both react in this way, their behavior becomes perfectly revealing. A's willingness to buy infoms B that she received good news; combining this information with his previous information, he discovers the true state. Her train of though is similar. Hence, the state becomes commonly known, and the only price at which they could trade is one at which they are indifferent to do so. Summing up, if it is commonly known that there is no reason to trade besides private information (speculation), then in equilibrium (almost) all information is transmitted - by actions or prices, depending on the trading environment - thereby eliminating the only reason to trade.

Subsequent research has shown this no-trade result to be fairly robust even to the absence of common knowledge of rationality and other troublesome assumptions, i.e., even if there is no information revelation. Notably, Fudenberg and Levine (2005) show that the no trade result also obtains in self-confirming equilibria (as defined in Fudenberg and Levine 1993) and marginal best response distributions (as defined in Fudenberg and Levine 1995). These equilibrium concepts are weakenings of the Nash equilibrium concept, particularly regarding the information players have about the game. In a self confirming equilibrium players might be wrong about counterfactuals. On the other hand, a broad class of learning procedures studied in the literature leads to stochastic outcomes of the game that have the marginal best response distribution property. ${ }^{7}$ Neither of these

\footnotetext{
${ }^{7}$ In a marginal best response distribution, each player might be wrong about his opponent's strategies, but gets an expected payoff of at least what he would get by playing a best response to the marginal distribution over his opponent's actions.
} 
concepts involve common knowledge of rationality or of actions. The basic idea behind the result is that any sensible learning procedure would guarantee at least the minmax payoff to each player, ${ }^{8}$ and since consuming the endowment (i.e., not trading) is feasible, and the endowment is efficient (since there are no reasons to trade prior to the arrival of information), the minmax payoff coincides with the utility of the endowment.

Heifetz, Meier and Schipper (2005) show an example, however, where two traders agree to trade based on private information. This can occur because each of them is unaware of the fact that her trading partner also possesses information, and furthermore, each holds a theory of her opponent's behavior which is consistent with what she observes.

In the previous example, suppose individual A not only is privately informed about $p$ but also resides in a space where only $p$ can be expressed - and hence is unaware of $q$. Similarly, individual B resides in a space where only $q$ is expressible, and is unaware of $p$. If $[p \wedge q]$ obtains, A thinks that the price may drop by $20 . \mathrm{B}$, on the other hand, thinks the price may rise by 20 . Moreover, in A's world there are only two possibilities: either B knows the same as A, or knows nothing. Within those possibilities, B's willingness to trade tells A that B knows nothing, and consequently cannot learn something useful for predicting the asset's value from his behavior. B thinks in a similar way about $\mathrm{A}$, and is therefore willing to buy upon good news. Hence, they both agree to trade.

A full blown model of speculation in asset markets is yet to be developed. This example shows, however, that HMS's model is capable of describing speculative trade.

\subsubsection{Incomplete contracts}

Two parties might want to promise each other transfers of money or goods contingent upon some events. An employer might want to delegate some tasks on an employee, and being unable to foresee the particular conditions her business will face in the future, she might wish to write down a contract that specifies what her employee should do in each situation, and how to react if he does not fulfill his part of the contract. Under omniscience, the best contract is a complete one, in the sense that it specifies for each conceivable situation what each party should do.

However, it is often (if not always) the case that the parties entering into a contract are unaware of some fact that, when revealed, leads them to revise their previous decisions. The influence of unforeseen contingencies on contracts is the subject of Incomplete Contract Theory (Hart and Moore, 1988). However, since this theory is based on the standard state space model, it cannot really portrait unforeseen contingencies; instead, the theory uses a modeling shortcut, namely it assumes that the parties can only bargain over a variable related to but different from their variable of interest. Unforeseen contingencies are thus relegated to the

${ }^{8}$ That is, it would be " $\varepsilon$-safe" in the terminology of Fudenberg and Levine (1995). 
motivation. Conceptual problems arise from this fact: as Maskin and Tirole (1999) suggest, the parties could write down a contract based on payoffs rather than external events or states, replicating what could be obtained by a complete contract.

Consider briefly what the explicit modeling of unawareness along the lines of HMS could mean for contract theory. The purpose of a contract is to engage in a transaction that otherwise would be infeasible because after the fact it may require the parties to do something they don't want. After the fire, the insurance company would certainly prefer not to send the check to its client, and a contract enforced by a legal system would allow it to promise an otherwise incredible act. The contract is thus a vehicle to commit.

An omniscient party would take care of his interest in all contingencies, leaving no detail aside. But under unawareness, this cannot be so: evidently the parties involved cannot write a contract that considers situations they both are unaware of.

Besides this obvious source of incompleteness, there is another of strategic nature. If the contract itself may generate awareness of certain possibilities, a party who is aware of a possibility and that believes his counterparty unaware of, may not wish to bring the subject up in the bargaining process in order to maintain his counterparty's current state of mind. For instance, the seller of a house does not want to make the contract contingent on the fulfillment of the city's plans to build a nuclear plant nearby. While the buyer may associate some truth to the proposition "my opponent might know something I don't," this fact does not allow her to make any inferences if the specific possibilities do not cross her mind. Contract incompleteness could then build itself up.

\section{Probability and Ambiguity}

The EU model puts on top of the standard state space model a probability that represents the DM's beliefs about the events in $2^{\Omega}$, the set that contains the events associated to his doubts or uncertainties. His behavior is obtained from the maximization of the expected value of the Bernoulli function, where the expectation is taken with respect to those beliefs as in Equation 1.

Ellsberg (1961) suggested two thought experiments where most individuals would behave in a way that it is impossible to rationalize with the EU model, and subsequent experimental work proved him right.

There are many versions of the paradox, but all of them are variations on the following idea. Consider an individual who is presented two urns (A and B) with 100 chips each, either red $(r)$ or blue $(b)$. The individual is told that urn A contains exactly 50 red and 50 blue chips, and that urn B also contains only red and blue chips, but he is not told in which proportions. The individual is asked to bet on a color: one chip is extracted, and if it matches the color of his choosing, he wins a prize of value $x$. If the color is different, he gets nothing. Before betting on the color, however, he is asked to choose the urn from which the chip will be extracted. 
Most individuals in experiments of this sort say they do not care which color they bet on, but prefer to bet on urn A, the one with the known composition. This behavior is inconsistent with the EU model. To see this, let us compute the expected utilities of each alternative. The following list indicates the expected utility of betting on urns $\mathrm{A}$ and $\mathrm{B}$ and colors $r$ and $b$ :

$$
\begin{aligned}
& E[u](A ; r)=\pi\left(r_{A}\right) u(x)+\pi\left(b_{A}\right) u(0) \\
& E[u](A ; b)=\pi\left(r_{A}\right) u(0)+\pi\left(b_{A}\right) u(x) \\
& E[u](B ; r)=\pi\left(r_{B}\right) u(x)+\pi\left(b_{B}\right) u(0) \\
& E[u](B ; b)=\pi\left(r_{B}\right) u(0)+\pi\left(b_{B}\right) u(x)
\end{aligned}
$$

where $u(\cdot)$ is the Bernoulli function and $\pi$ the probability; in turn, $r_{A}$ denotes the event of extracting a red chip from urn A.

If the individual is indifferent to the colors in both urns, he must associate a $50 \%$ chance to obtaining each color from either urn:

$$
E[u](A ; r)=E[u](A ; b) \Rightarrow \pi\left(r_{A}\right)=\pi\left(b_{A}\right)=\frac{1}{2}
$$

$$
E[u](B ; r)=E[u](B ; b) \Rightarrow \pi\left(r_{B}\right)=\pi\left(b_{B}\right)=\frac{1}{2}
$$

This implies, however, that he must associate the same utility level to both urns:

$$
E[u](A)=\frac{1}{2}[u(x)+u(0)]=E[u](B)
$$

This is to say, he must be indifferent among urns. Taken from a different perspective, indifference to colors in A means:

$$
E[u](A)=\frac{1}{2}[u(x)+u(0)]
$$

Meanwhile, the utility of choosing urn B is given by:

$$
E[u](B)=\max \left\{\pi\left(r_{B}\right) u(x)+\pi\left(b_{B}\right) u(0) ; \pi\left(r_{B}\right) u(0)+\pi\left(b_{B}\right) u(x)\right\}
$$

so that if $\pi\left(r_{B}\right)<\frac{1}{2}$, the individual would prefer to bet on blue and

$$
E[u](B)=\pi\left(r_{B}\right) u(0)+\pi\left(b_{B}\right) u(x)>\frac{1}{2}[u(x)+u(0)]=E[u](A)
$$


If $\pi\left(r_{B}\right)>\frac{1}{2}$, the individual would rather bet on red but still on urn B, using the same argument. Therefore, there is no scenario we can think of in which urn $A$ is preferred to urn $B$ if the individual associates a $50 \%$ chance with each color in A. If he is indifferent to colors in B, he must be indifferent to which urn is used. If he is not indifferent to the colors in B, then he must prefer B.

Hence, if an EU-maximizer is indifferent to the colors in both urns, he must be indifferent to which urn is used, a prediction systematically contradicted by experimental data (Camerer, 1995, pg. 646, surveys some of this experimental work).

There could be many ways of amending the model so it is capable of accommodating this behavior. One such possibility, and the one we explore here, is to abandon probabilities to represent beliefs. This road though begs the question of how probabilities got into expected utility in the first place. The way one rationalizes the abandonment of probabilities depends on how one justified its use, which in turn depends on how "probability" is interpreted. There are essentially two extreme views on this matter: objectivism and subjectivism. ${ }^{9}$

Objectivism holds that a probability is a characteristic of an object not unlike weight or mass, and that is therefore amenable to scientific study. Under an objectivist interpretation, EUT portrays a very knowledgeable DM, one that has learned the probabilities of all relevant events. Ellsberg's experiment can be thought of as a departure from that assumption, and the question that opens is how the theory can be extended to situations in which the DM does not know the relevant probabilities. ${ }^{10}$

Subjectivism, on the other hand, holds that probabilities are a convenient description of the state of ignorance of any given person, and that what they represent does not exist outside the subject's mind. From this perspective, it is an open question whether an individual's beliefs -which are indirectly observable from his behavior- can be represented by a probability. If this cannot be done, then the theory must be expanded to better describe the subject's behavior.

The literature on "ambiguity" covers both interpretations. From a modeling perspective, it essentially replaces the probability with either a capacity (the Choquet expected utility model, henceforth the CEU model) or a set of probabilities (the Maxmin Expected Utility Model, henceforth MEU). To understand what this means, recall that a probability is simply a real-valued function that assigns a number

\footnotetext{
${ }^{9} \mathrm{~A}$ more detailed account of the different ways of interpreting probability and their implications for EUT and its applications is deferred until Section 4.4.

${ }^{10}$ Such ignorance of the relevant probabilities is often referred to as "Knightian uncertainty", after Frank Knight's (1921) distinction between risk (outcome unknown but with known probabilities) and uncertainty (outcome and probabilities unknown).
} 
between 0 and 1 to each event included in a particular collection called algebra, ${ }^{11}$ with the following properties:

$$
\begin{aligned}
& \text { ( } \pi 1) \quad \pi(\varnothing)=0, \quad \pi(\Omega)=1 \quad \text { (normalization) } \\
& (\pi 2) \quad E \subseteq E^{\prime} \Rightarrow \pi(E) \leq \pi\left(E^{\prime}\right) \quad \forall E, E^{\prime} \in \mathfrak{M} \quad \text { (monotonicity) } \\
& \text { ( } \pi 3) \quad \pi\left(E \cup E^{\prime}\right)=\pi(E)+\pi\left(E^{\prime}\right)-\pi\left(E \cap E^{\prime}\right) \quad \text { (additivity) }
\end{aligned}
$$

In turn, a capacity is a not-necessarily additive probability, i.e., it is a realvalued function on the algebra that only satisfies $\pi 1$ and $\pi 2$. As such, it is a generalization of the notion of probability.

The following distinctions will be useful in the sequel. If for all events in the algebra it is true that $\pi\left(E \cup E^{\prime}\right) \leq \pi(E)+\pi\left(E^{\prime}\right)-\pi\left(E \cap E^{\prime}\right)$, then the capacity is said to be convex. It is said to be concave if the inequality is reversed. Note though that a capacity may be neither convex nor concave.

\subsection{Modeling ambiguity}

This section deals primarily with the Choquet Expected Utility model (henceforth, CEU.) The chief proposal is to consider capacities to represent beliefs, thus giving rise to an expected utility representation of preferences where the probabilities are not additive. MEU is only briefly mentioned towards the end.

The CEU model was pioneered by Schmeidler (1989). In the context of Ellsberg's paradox, Schmeidler's observation is that we may disassociate indifference to colors - no reason to prefer one color over another - from indifference to urns. Instead of associating a "probability" to each event, let us say that the individual associates a degree of confidence to the occurrence of a state, $v$, not necessarily represented by a probability. In particular, rewrite Equation 5 as:

${ }^{11}$ An algebra $\mathfrak{M}$ is a set of events, with the properties of being closed under complementation and union, that is if two events $E$ and $E^{\prime}$ are in the algebra, then both their complements and their union also are in it:

(A1) $\varnothing, \Omega \in \mathfrak{M}$

(A2) $E \in \mathfrak{M} \Rightarrow E^{c} \in \mathfrak{M}$

(A3) $E, E^{\prime} \in \mathfrak{M} \Rightarrow E \cup E^{\prime} \in \mathfrak{M}$

These requirements are natural. From a syntactic perspective, they amount to demand that if one can talk about a given proposition, one should also be able to talk about its negation (A2); that there exists a proposition that is trivially true, or a tautology, and also its negation, a contradiction (A1); and that one can compound the available propositions to form new ones, with the logical connective "or" (A3) and "and" by appealing to the de Morgan's laws, for $\left.E \cap E^{\prime}=\left(E^{c} \cup E^{*}\right)^{c}\right)$. Hence, if $p \equiv$ "it will rain tomorrow" and $q \equiv$ "the apple is rotten" are expressible in the model (i.e., $[p],[q] \in \mathfrak{M}$ ), then "it will not rain tomorrow", "the apple is not rotten", "the apple is either rotten or not rotten", "it will not rain tomorrow but the apple is rotten", etc, also are. $2^{\Omega}$ is an example of an algebra; in fact, it is the largest one that can be formed from $\Omega$. 


$$
\begin{aligned}
& E[u](A ; r)=v\left(r_{A}\right) u(x)+\left(1-v\left(r_{A}\right)\right) u(0) \\
& E[u](A ; b)=\left(1-v\left(b_{A}\right)\right) u(0)+v\left(b_{A}\right) u(x) \\
& E[u](B ; r)=v\left(r_{B}\right) u(x)+\left(1-v\left(r_{B}\right)\right) u(0) \\
& E[u](B ; b)=\left(1-v\left(b_{B}\right)\right) u(0)+v\left(b_{B}\right) u(x)
\end{aligned}
$$

Indifference to colors implies:

$$
\begin{aligned}
& E[u](A ; r)=E[u](A ; b) \Rightarrow v\left(r_{A}\right)=v\left(b_{A}\right) \\
& E[u](B ; r)=E[u](B ; b) \Rightarrow v\left(r_{B}\right)=v\left(b_{B}\right)
\end{aligned}
$$

However, urn $\mathrm{A}$ is preferred to urn $\mathrm{B}$ as long as $v\left(r_{A}\right)>v\left(r_{B}\right)$ :

$$
E[u](A)=v\left(r_{A}\right) u(x)+\left(1-v\left(r_{A}\right)\right) u(0)
$$

$$
>E[u](B)=v\left(r_{B}\right) u(x)+\left(1-v\left(r_{B}\right)\right) u(0)
$$

The capacity $v(\bullet)$ evaluates states, but unlike a probability, $v\left(r_{B}\right)+v\left(b_{B}\right) \neq 1$, that is, the belief is not additive. Surely colors red and blue are the only possibilities for urn $\mathrm{B}$, but this is expressed on $v\left(\left\{r_{B}, b_{B}\right\}\right)=1$ rather than $v\left(r_{B}\right)+v\left(b_{B}\right)=1$.

If the capacity is convex $\left(v\left(r_{A}\right)+v\left(r_{B}\right)<1\right)$ then the Choquet expected value of the Bernoulli function assigns all the unassigned weight $\left(1-v\left(r_{A}\right)-v\left(r_{B}\right)\right)$ to the worst-case scenario, resulting in a lower utility level than betting on the unambiguous urn A, rationalizing the paradox. Urn A represents a less ambiguous choice than urn $\mathrm{B}$, because the individual has more information and hence more confidence in his beliefs, even though he has not reason to believe that one color is more likely than the other for either urn.

A convex capacity thus leads to a certain form of pessimistic behavior, and is usually interpreted as reflecting uncertainty aversion. A concave capacity does the opposite: all weight exceeding 1 is reassigned from the worst case scenario to the best case scenario, resulting in optimistic behavior or ambiguity love.

Schmeidler (1989) identifies a set of properties for individual behavior that imply an EU-representation of preferences (see below), but where the expectation is not taken over a probability but over a capacity. The lack of additivity, however, implies that the usual integral cannot be applied here. The appropriate integral concept is that of Choquet (which explains the name of the model). If $C(a)=\left\{c_{1}, c_{2}, \ldots, c_{n}\right\}$ is the set of possible consequences under act $a$, where $u\left(c_{i}\right) \geq u\left(c_{i+l}\right)$ for all $i$, then the Choquet integral is given by: 


$$
\int u(f) d v=\sum_{i=l}^{n-l}\left[u\left(c_{i}-u\left(c_{i+l}\right)\right)\right] v\left(\bigcup_{j=l}^{i} A_{j}\right)+u\left(c_{n}\right)
$$

where $A_{j}$ is the event in which consequence $c_{j}$ obtains under act $a$. In the twostate urn example that introduced this section, this definition means that as long as $v\left(r_{\bullet}\right)+v\left(b_{\bullet}\right)<1$, the higher-utility scenario is weighted by $v(\bullet)$ and the lowerutility scenario by $I-v(\bullet)$, that is, the capacity associated to this state plus all nonassigned weight, as asserted before.

More generally, when there are two states the indifference curves over risky consumption profiles are kinked (and hence non differentiable) at the certainty line. For instance, let $\Omega=\left\{\omega_{l}, \omega_{2}\right\}$ be the state space and the set of consequences $\mathfrak{R}^{2}$, that is, ordered pairs of positive reals. Then, each act is a bundle $\left(c_{1}, c_{2}\right)$ indicating a consumption level for each state. Suppose beliefs have the form given by Table 12a.

TABLE 12.a A CONVEX CAPACITY

\begin{tabular}{c|c}
$E$ & $v(E)$ \\
\hline$\varnothing$ & 0 \\
$\left\{\omega_{1}\right\}$ & $\frac{1}{4}$ \\
$\left\{\omega_{2}\right\}$ & $\frac{1}{4}$ \\
$\left\{\omega_{1}, \omega_{2}\right\}$ & 1
\end{tabular}

TABLE 12.b A CONCAVE CAPACITY

\begin{tabular}{c|c}
$E$ & $v(E)$ \\
\hline$\varnothing$ & 0 \\
$\left\{\omega_{1}\right\}$ & $\frac{3}{4}$ \\
$\left\{\omega_{2}\right\}$ & $\frac{3}{4}$ \\
$\left\{\omega_{1}, \omega_{2}\right\}$ & 1
\end{tabular}

Then, if an act $a$ is such that leads to $c_{1}>c_{2}$,

$\int u(c) d v=\frac{1}{4} u\left(c_{1}\right)+\frac{3}{4} u\left(c_{2}\right)$

whereas in the opposite case, when $c_{1}<c_{2}$

$\int u(c) d v=\frac{3}{4} u\left(c_{1}\right)+\frac{1}{4} u\left(c_{2}\right)$ 
PICTURE 1

AN INDIFFERENCE CURVE UNDER AMBIGUITY AVERSION

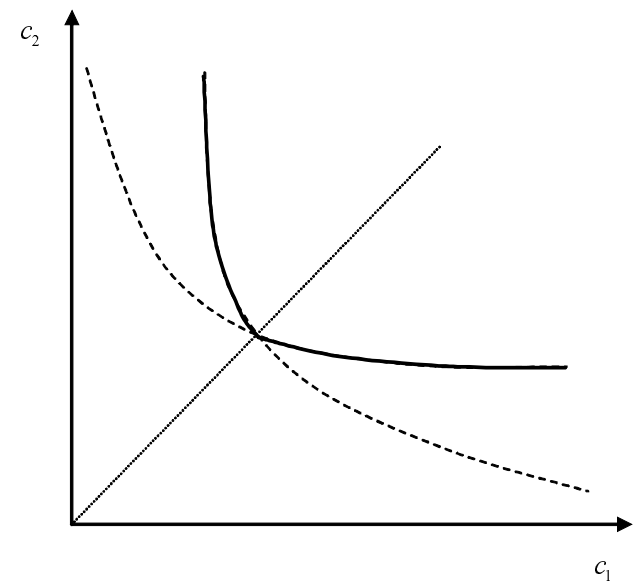

The Choquet integral, then, adds the unassigned weight of a convex capacity $l-v(E)-v\left(E^{c}\right)$ to the worst possible outcome. The certainty line separates the cases where state $\omega_{1}$ is associated to the worst outcome from the cases where it is state $\omega_{2}$. The indifference curve then consists of the union of two pieces of regular EU functions' at each side of the certainty line, the indifference curve coincides with that of the more pessimistic EU function. Picture 1 depicts this; the CEU indifference curve is the bold curve, formed from the two regular EU indifference curves under consideration (dotted). Observe that the limiting case of a CEU function, that of maximum ambiguity (where for all states, $v(\omega)=0$, corresponds to $\min \left\{c_{1}, c_{2}\right\}$. Thus, in the limiting case the DM evaluates the available acts according to their worse-case scenarios.

On the other hand, if the capacity is concave the "extra" weight (the excess over 1) is assigned to the best possible outcome, that is, precisely the opposite of the convex-capacity. In the case of the capacity of Table 12.b, the CEU is given by:

$$
\int u(f) d v= \begin{cases}\frac{3}{4} u\left(c_{1}\right)+\frac{1}{4} u\left(c_{2}\right) & \text { if } c_{1}>c_{2} \\ \frac{1}{4} u\left(c_{1}\right)+\frac{3}{4} u\left(c_{2}\right) & \text { otherwise }\end{cases}
$$

Intuitively, the former is being "optimistic", while the latter "pessimistic." Recall that the form of the capacity is obtained by looking at the DM's behavior, so beliefs are not really separable from attitudes towards ambiguity, as in the attitudes towards risk case. For that reason, a DM with a convex capacity is called "ambiguity averse" (and the degree of his aversion measured by the difference $\left.1-\left[v(A)+v\left(A^{c}\right)\right]\right)$, while a DM with a concave capacity is called an "ambiguity lover", paralleling the terminology employed for risk aversion or love. 
A very interesting feature of this model is that it is capable of rationalizing puzzling behavior beyond Ellsberg's thought experiments. Consider for instance the case of a person who bets heavily at a casino and at the same time buys life and health insurance. While a risk-averse EU-maximizer is risk-averse in every dimension, a CEU-maximizer need not hold equally non-additive beliefs regarding every uncertainty. Thus, the same individual can have non-additive beliefs with respect to one variable and additive with respect to another.

Imagine a person who entertains doubts about the following propositions in connection to the next day: $p=$ "it will rain" and $q=$ "there will be an earthquake", defining four states as in Table 1 . The capacity of Table 13 is additive with regard to the possibility of rain, but non-additive with regard to the possibility of an earthquake (the 3 -state events are omitted for brevity):

TABLE 13

A CAPACITY THAT IS NON-ADDITIVE IN ONE DIMENSION WHILE ADDITIVE IN THE OTHER

\begin{tabular}{c|c}
$E$ & $v(E)$ \\
\hline$\varnothing$ & 0 \\
{$[p \wedge q]$} & 0.07 \\
{$[p \wedge \neg q]$} & 0.1 \\
{$[\neg p \wedge q]$} & 0.03 \\
{$[\neg p \wedge \neg q]$} & 0.1
\end{tabular}

\begin{tabular}{c|c}
$E$ & $v(E)$ \\
\hline$[p]$ & 0.7 \\
{$[\neg p]$} & 0.3 \\
{$[q]$} & 0.2 \\
{$[\neg q]$} & 0.3 \\
$\Omega$ & 1
\end{tabular}

In this example, the individual associates a probability of $70 \%$ to rain and $30 \%$ to not rain, and therefore the DM will behave as an EU-maximizer if faced with decisions whose consequences depend exclusively on whether it will rain or not. However, his degree of confidence in an earthquake happening is $20 \%$, it not happening $30 \%$, and hence he will behave as a CEU-maximizer in decisions involving earthquake/no earthquake as an event. The remaining $50 \%$ can be interpreted, as remarked earlier, as the degree to which the situation seems ambiguous to him. While this person would behave very much like an extremely risk averse individual in situations that depend on the occurrence of an earthquake, he will not do so in situations that depend on the possibility rain. Hence, the generalization presented by CEU is non-trivial. One cannot just say "the DM perception is ambiguous"; one should also specify where is that ambiguity, i.e., which variables are affected by it.

There is a second popular model that rationalizes Ellsberg's paradox: the Maxmin Expected Utility model (sometimes also referred to as the Multiple Prior model), due to Gilboa and Schmeidler (1989). This model posits that the DM does not think about one but many probabilities; for instance, in Ellsberg's experiment the individual may think the probability of extracting a red chip from urn B is anything between $1 / 4$ and $3 / 4$. According to this model, the individual evaluates an 
act according to the worst possible case among those probabilities, ${ }^{12}$ and chooses what the best act so evaluated, resulting in a utility function of the form:

$U(\bullet)= \begin{cases}\min _{\pi\left(r_{B}\right) \in\left[\frac{1}{4}, \frac{3}{4}\right]} E[u](B)=\pi\left(r_{B}\right) u(x)+\left(1-\pi\left(r_{B}\right)\right) u(0) & \text { if } a=\text { bet on red } \\ \min _{\left(1-\pi\left(r_{B}\right)\right) \in\left[\frac{1}{4}, \frac{3}{4}\right]} E[u](B)=\pi\left(r_{B}\right) u(0)+\left(1-\pi\left(r_{B}\right)\right) u(x) & \text { if } a=\text { bet on blue }\end{cases}$

which corresponds to:

$$
U(a)= \begin{cases}\frac{1}{4} u(x)+\frac{3}{4} u(0) & \text { if bet on red } \\ \frac{3}{4} u(0)+\frac{1}{4} u(0) & \text { if bet on blue }\end{cases}
$$

The preference relation over acts is therefore representable by the following function:

$$
\min _{\pi \in \Pi} E_{\pi}[u(c) \mid a]
$$

This function is maximized over acts: $\max _{a \in A} \min _{\pi \in \Pi} E_{\pi}[u(c)]$, hence the maxmin name.

Observe that the resulting function in Equation 17 is exactly as that of Equation 15. It turns out that this model coincides exactly with the CEU model if the associated capacity is convex and the set of probabilities is such that for all events $\pi(E) \geq v(E)$ (Gilboa and Schmeidler, 1993). Their limiting case, as ambiguity grows large, must also coincide: extreme ambiguity aversion leads to the worsecase scenario decision rule, i.e., each act is evaluated according to its worst possible consequence. In general, then, either model can be used to characterize ambiguity averse behavior.

We turn now to the theoretical underpinnings of these models.

\subsection{Objective Probability}

Von Neumann and Morgenstern (1946) define an act $a \in A$ as a probability distribution over consequences, or lottery. For instance, if a DM is invited to bet on the outcome of a coin toss, von Neumann and Morgenstern would characterize the decision problem as composed of two mutually exclusive acts, described by:

$$
\begin{array}{ll}
\text { Accepting: } & \operatorname{Pr}(\text { winning } 1,000)=\operatorname{Pr}(\text { losing 100 })=\frac{1}{2} \\
\text { Rejecting: } & \operatorname{Pr}(\text { winning } 1,000)=\operatorname{Pr}(\operatorname{losing} 100)=0
\end{array}
$$

${ }^{12}$ No to be confused with the worst possible outcome, i.e., the worst conceivable consequence of an act. Yet, see below. 
Thus, the probability is part of the description of the possibilities an individual faces, the courses of action open to him. Using this concept, the most natural interpretation of probability is of an objective nature: the probability exists outside the DM. Since in any decision-making problem, the DM must know the alternatives from which he must choose, it follows that he must know the probabilities of each event. Depending on the application, this assumption may be too strong, and yet it is unavoidable if acts are identified with lotteries from the start.

Von Neumann and Morgenstern assume the DM can establish an order of preference among all acts (i.e., a preference relation), and impose two conditions over that preference. Consider a reference lottery that yields a better outcome than any other act under consideration with a given probability, and another outcome that is worse than any other act under consideration with the complementary probability. The conditions are then:

(O1) For each act there is always a probability of winning the reference lottery that would make the DM indifferent between the act and the lottery; and

(O2) If act $a$ is preferred to act $b$, then a lottery that with some probability leads to $a$ and with the complementary probability to $c-$ a third, unspecified act-, must be preferred to another lottery obtained from $b$ transformed in the same way.

Condition $(\mathrm{O} 1)$ is a continuity assumption. Condition (O2) imposes that there can be no complementarities among consequences: compounding the two options above in a lottery with a third one does not alter the order of preferences. These assumptions lead to an EU representation of preferences.

If an act is not a lottery over consequences but a set of them, then the previous axioms are not appropriate because they don't apply to the new objects of choice. Ahn (2005) obtains a maxmin representation of preferences defined over sets of lotteries by assuming that (O1') preferences are continuous, that (O2') enlarging a set of lotteries makes it (weakly) less desirable, and that (O3) if two sets of lotteries are each preferred to a third, then their union is also preferred to that third set. Observe that condition (O2') embeds ambiguity aversion: if the ambiguity increases by introducing the possibility of an even worse lottery than previously considered, the DM is worse off even though the possibilities now may also include better lotteries than before: the worse lotteries are more important for her.

\subsection{Subjective Probability}

The main difference between the approaches of von Neumann and Morgenstern, and Savage (1957) is their distinctive way of describing acts. While for von Neumann and Morgenstern an act is a probability distribution over consequences in itself, for Savage it is merely a map from states to consequences, deprived of any probability judgments. In this view, a DM understands an act as a list of possible consequences, one for each possible state of the world: $a: \Omega \rightarrow C$. 
Continuing with the example of the bet on the outcome of a coin toss, Savage would describe the two available acts by:

Accepting: win 1,000 if heads, lose 100 otherwise

Rejecting: win/lose nothing in either case

The most striking aspect of Savage's theory is that he manages to identify a set of behavioral assumptions that lead to an expected utility representation of preferences, involving a probability function -which can be interpreted as the DM's implied (as opposed to declared or known) beliefs- and which, moreover, satisfies all properties of probabilities.

The significance of this fact is twofold. First, if one is willing to assume that an individual's behavior obeys these properties (see below), then the existence of a probability function, to be interpreted as the DM's implicit beliefs, is granted. That is, the idea that an individual's beliefs are representable by a probability is not an assumption but a conclusion. The implied probability is therefore individual-specific, and hence subjective. Second, its existence does not require postulating anything about the DM's knowledge about empirical frequencies or the like.

Contrary to our maintained assumption, Savage's axiomatization requires $\Omega$ to be infinitely dimensional and is mathematically more intricate. Our discussion of Savage's theory will therefore follow Gul's (1992) adaptation to finite state spaces.

The starting point is a continuous ${ }^{13}$ preference relation over acts. An event $E$ will be called null if the individual is indifferent between any two acts that coincide in $E^{c}$, the idea being that the individual considers $E$ to be irrelevant. The preference relation is required to satisfy the following properties:

(S1) If an act leads state-by-state to a better outcome than a second act, then it must be preferred to the latter.

(S2) Consider three acts $a, b$, and $c$, and one non-null event $E$; create two new acts $a^{\prime}$ and $b^{\prime}$ by replacing in the states that belong to $E^{c}$ all consequences of $a$ and $b$ by those of $c$. Then, if the individual prefers $a$ to $b$ it must be the case that he also prefers $a^{\prime}$ to $b^{\prime}$, and vice versa.

(S3) There exist an event $E$ such that for any two consequences the individual is indifferent between an act that leads to the first consequence upon event $E$ and to the second upon its complement, and an act that leads to the second consequence upon event $E$ and to the first upon its complement.

\footnotetext{
${ }^{13} \mathrm{~A}$ preference relation is said to be continuous if for each act the set of acts that are weakly preferred to it and the set of acts that are weakly dispreferred to it are both closed. This assumption is necessary for guaranteeing the existence of a utility function that represents the preference relation in the first place, regardless of whether one considers a decision problem under certainty or under uncertainty.
} 
(S1) is a consequentialism assumption: acts are evaluated by their consequences. (S2) is similar to $(\mathrm{O} 2)$. (S3) will ultimately require that the state space can be partitioned into two equally likely events. These assumptions are sufficient to guarantee the existence of a (unique) probability over $2^{\Omega}$ and a Bernoulli function whose expectation with respect to that probability represents the preference relation over acts (for the proof, see Gul 1992).

The contrapositive to this representation theorem says that if the observed behavior cannot be rationalized by an EU function, then it does not obey the above properties. As a consequence, Ellsberg's paradox urges a subjectivist to revise those axioms.

Schmeidler's work (1989) fits precisely along those lines. ${ }^{14}$ The basic idea is to relax S2 so it holds not for all acts but only for those that are comonotonic. Two acts are comonotonic if they both generate the same ordering of states in the sense of ex post preference (i.e., over consequences); this is to say, if state 1 is good and state 2 is bad under act $a$, then state 1 must be also be better than state 2 under act $b$ for $a$ and $b$ to be called comonotonic.

\subsection{What Do We Mean by Probability?}

We just saw that there are different EU theories, depending on what does one mean by "probability". It is then necessary to discuss the available interpretations in some length ${ }^{15}$ in order to grasp more fully what EU is, and what its proposed amendments mean.

\subsubsection{Objective views}

Objective views of probability hold that "probability" is a characteristic of the object under consideration, like weight, mass or temperature, and not of the observer. Presumably, the probability of an event can be learned through scientific examination, be it by empirical research or introspective reasoning.

The idea that probabilities are objective leads immediately to questions about the validity of EU theory as a positive theory. To be sustained, the theory needs to explain how these probabilities are "revealed" or "known" to decision makers.

The significance of this for EUT is that it severely limits its applicability to situations in which such probabilities can be computed. Several authors have argued against the possibility of applying this (or even any) mathematical model to explain behavior under uncertainty. In his General Theory, for example, Keynes distinguished between long-run and short-run expectation: the former referred to

${ }^{14}$ In truth, Schmeidler's (1989) work was not carried out on a purely subjective framework. However, Nakamura (1990) extended it to such framework and, moreover, using a finite state space. MEU can also be founded on a modified Savage framework, along the lines of CasadesusMasanell et al. (2000).

${ }^{15} \mathrm{~A}$ deeper and more complete discussion of probability can be found in Hájek (2002). 
situations in which individuals can accumulate too little evidence to base judgments on it, thereby rendering probability assessments impossible. Knight, on the other hand, is credited for his distinction between risk (a situation in which the DM cannot predict the consequence but knows the probabilities) and uncertainty (where the latter knowledge is also absent), obviously holding an objective view.

Hence, from the perspective of EUT, objective views suffer from the common drawback that it cannot be taken as a universal, positive theory of human behavior, for in many, perhaps most, instances the relevant probabilities cannot be assumed to be known. This fact is of extraordinary importance and often overlooked.

Friedman's (1953) epistemological view, however, provides a partial reconciliation of objective views and universal applicability: the model must be judged by its predictions and not by its assumptions. The trouble is that from one model many predictions are drawn. If the researcher focuses on one prediction, then this may work. However, the discipline constantly seeks a general model that provides particular predictions. For instance, if the CAPM is taken to predict that expected returns are linearly related to their "betas", it may be of no consequence to assume that all investors "know" the joint distribution that characterizes asset returns. If it is taken to predict that all investors will hold a market portfolio, however, one is forced to look at the assumptions with more suspicion.

At any rate, universality may be the main practical reason behind why many economists declare their support for the subjectivist view. However, in many applications of EU theory in economics it is easy to recognize some, and often much, objectivism, a notable example being the Theory of Rational Expectations. A quick review of the most common objective interpretations follows.

Generally speaking the classical interpretation of probability holds that a DM should list all possibilities, consider each of them as "equally likely," and then deduce the probability of each event by "counting" the different ways that such event may occur. The probability of an event is thus defined by the ratio "number of states consistent with the occurrence of the event/total number of states".

For instance, consider a gamble where a woman flips a coin once. If the outcome is heads, she pays $\$ 2$ to a bettor. If tails, she flips it again. If the outcome of the second trial is heads, she pays $\$ 2^{2}$ to the bettor. Otherwise she tosses it again, and so on. If, in the first $\mathrm{n}$ trials, the outcome was heads, the prize in case of tails in the $(\mathrm{n}+1)$ th trial is $\$ 2^{\mathrm{n}+1}$. According to the classical interpretation, every gambler in this situation should consider the probability of winning exactly $\$ 2^{n}$ to be $\left(\frac{1}{2}\right)^{n}$. This conclusion is arrived at in the following way: "The number of outcomes in each trial is 2: the coin either comes up heads or tails. Hence, in each trial, the probability of heads is $\left(\frac{1}{2}\right)$. Therefore, after $\mathrm{n}$ trials, the possible outcomes take the form (x heads, $\mathrm{n}-\mathrm{x}$ tails), and for each of these, there are $\left(\begin{array}{l}n \\ x\end{array}\right)$ sequences of heads-tails consistent with such outcome. However, the game ends the first time a tails appears. It follows that to win exactly $\$ 2^{n}$, in the first (n-1) trial the outcome has to be heads and in the last one, tails. The probability of that is clearly $\left(\frac{1}{2}\right)^{n}$." 
Possibly the earliest construction of EU is that of Bernoulli (1738), who proposed it as a solution to the Saint Petersburg paradox, one of the early discussions on the notion of value. The Saint Petersburg paradox used a gamble like the above to say that such a gamble has an infinite expected payoff, but clearly no one should be willing to pay even finite amounts such as, say, $\$ 1,000,000$. In effect, if $c(n)$ is the consequence of obtaining tails for the first time in trial $n$,

$$
E[c(n)]=\sum_{n=0}^{\infty}\left(\frac{1}{2}\right)^{n} 2^{n}=\sum_{n=0}^{\infty} 1
$$

which does not converge. Bernoulli, then, proposed the following "solution": if the gambler does not evaluate the state according to the prize he obtains, but rather according to a function $u(c(n))$, then he will be willing to pay a finite amount, as long as this function is concave. For instance, if $u(c)=\sqrt{c}$,

$$
E[u(c(n))]=\sum_{n=0}^{\infty}\left(\frac{1}{2}\right)^{n} \sqrt{2^{n}} \approx 2.4,
$$

yielding a certainty equivalent of 5.76, a much more reasonable amount to pay for such a game. Of course, what Bernoulli constructed was the expected-utility function, giving simultaneously an argument for its use in decision theory (whether to pay an amount $x$ for the right to play) and for what later became known as risk aversion, represented by the concavity of the Bernoulli function. Interestingly, then, EUT was born of a classical, objective view of probability.

There are many objections to the classical view of probability. Possibly the most devastating is the fact that any problem admits more than one description of the set of states. Since every state carries the same probability, different descriptions lead to different probabilities and hence to different preferences over acts. To give an obvious example, if the possibility of the coin landing on its edge is considered, then the probability of winning exactly $\$ 2^{\mathrm{n}}$ should be $\left(\frac{1}{3}\right)^{n}$ instead of $\left(\frac{1}{2}\right)^{n}$. In the face of this, the theory does not uniquely predict what a DM would do.

The propensity view in some sense extends the notion of classical probability to allow for non-equal probability assignments, while retaining the idea that the probability is a characteristic of the object. Probability is then a deep, unknown parameter, which exists whether or not an observer is aware of its existence or value. Probability can be learned about by means of scientific investigation and the empiricist must experiment to discover its value. The deep parameter, however, can only be known precisely in an experiment that considers infinitely many repetitions.

Hence, a coin propends to exhibit a balanced number of heads and tails when tossed, in the same way a given light bulb propends to remain operational about 5,000 hours.

However, it is extremely unclear why these propensities must obey the properties usually attributed to probabilities (that is, normalization, monotonicity and additivity.) Each of these properties should in itself be the object of scientific 
inquiry. Yet, it is very hard to imagine a test for whether the limit of a sequence of experiments is the purported distribution.

On the other hand, the definition entails a great deal of vagueness. Since all experiments must consider finitely many repetitions, then some notion of closeness must be adopted. These notions take the form of a set of conventions among researchers, which are often hard to defend - consider the magical number 30 as the minimum number of observations in regression analysis or the $5 \%$ considered the acceptable level of significance in statistical inference.

A more basic objection refers to the assumption that the experiments were made under "similar conditions." In many cases one might be forced to accept that if the conditions were literally the same, one should expect to see the same outcome. If, for instance, the coin was flipped two times, with the same force being applied in the same point, etc., it should land in the same place, in the same position. If this is true, then saying that $50 \%$ of the times it lands heads up merely says that the conditions were not the same in all coin flips.

The trouble is perhaps that often attached to the propensity view is the idea that random events exist in nature: the outcome of a coin flip is random, as is the duration of a light bulb or the price of a financial asset. In many instances, whether random variables exist in nature or only are artifacts to represent the observer's ignorance is of no consequence in research. Yet accepting the former means that random variables cannot be used to represent processes we know or feel are not random. The example of the previous paragraph suggested that the outcomes of coin flips are deterministic, although hard for humans to predict. Consider a different example: a child who is learning basic mathematics could certainly entertain doubts when faced with the question, how much is 322 plus 25 ? He may entertain many possibilities and may not be confident of his answer. Can his choice of answer be modeled by a EU maximization? As the result of the sum is not really random, an adherent to the first view would say no: random variables are for random events. A subjectivist, on the other hand, would be happy to do so.

In general, questions like "what is the probability that candidate $\mathrm{X}$ wins in the next poll?" are meaningless under this view. EUT, hence, would not be applicable to situations where probabilities cannot be defined.

The frequentist view holds that probability refers to the frequency with which each particular event occurs, upon repeating an experiment under similar conditions a large number of times. It is in essence a mere, but convenient, description of the data.

As such, to the frequentist probability is not defined for one-shot experiments (or they are trivial: the probability of an outcome is either 0 or 1 , depending on the observed outcome). As a consequence, this view supports a narrow application of EUT, as did the previous one.

The logical view maintains that probability refers to the degree of truth of a proposition. Under this paradigm, probability is an extension of propositional logic, constructed over a syntactic model.

That such an extension can be satisfactorily made, and that it can be made by using probabilities, is a matter of controversy among logicians. But even 
assuming it can, it is not clear at all why individuals should base their behavior on these numerical values. For instance, a given proposition (event) may be considered to be more confirmed by evidence than another, and hence assigned a higher probability. Yet, EU requires the DM to behave differently when the ratio of these probabilities is, for example, 2 , than when it is 3 , although both cases conform equally to the notion that the first proposition is better confirmed by evidence than the second one. Put another way, the cardinality of probabilities is essential to decisions based on EU, although it is not necessarily so to represent degrees of confirmation.

\subsubsection{The subjective, personalistic, or Bayesian, view}

The subjective view of probability holds, on the other hand, that probability is a numerical representation of the degree of belief, or confidence, a DM has in a specific event occurring. The probability is thus a characteristic of the subject and not the object. It is an expression of his ignorance and doubts, in his present state of knowledge.

As such, it gains universality: every DM may hold beliefs and entertain doubts about many events, be they random or not, if one insists on the existence of random events. It is not necessary to assume that the DM has experience with "similar" problems or that he knows relevant empirical frequencies. The subjectivist holds that, nonetheless, every DM may have an assessment, and that that assessment can be written in the form of a probability. This is not to say that experience, knowledge or information is useless to making an assessment: the theory would eventually explain belief-change in the presence of information, and in general, reconcile it with objectivist views in situations where a large number of experiments can be performed. It just does not limit itself to this kind of situation.

Thus, a DM may have beliefs about who is going to win the 2011 presidential election, despite the fact that the candidates have not even been officially nominated. Moreover, these beliefs are assumed to exist (even though we cannot think of a frequency distribution created from a large number of similar experiments), to differ from the "favorable cases/possible cases" formula (which would not only assign the same probability to each possible candidate, namely $1 / 10,000,000$ in the case of Chile!), and to vary across people as well. Thus, besides its intuitive appeal and beautiful construction, the strong point of the subjective approach is its universal applicability to any decision problem. In the end, when faced with a decision problem, every DM makes a choice, so it is nice to have a theory that accounts for it or explains it.

The following informal summary of the meaning of "the probability of heads is $\frac{1}{2}$ " using the different interpretations may be useful: 
TABLE 14

THE MEANING OF THE PHRASE “THE PROBABILITY OF HEADS IS $\frac{1}{2}$ ”

View/School [Probability is] Meaning/Interpretation

Classical [A characteristic of the experiment of tossing a coin]

Heads is one of the two possible outcomes of the experiment with "tossing a coin."

Propensity [A characteristic of coins]

Coins propend to land heads up as much as they propend to land tails up when tossed.

Frequentist [Summary of evidence from many experiments]

Experiments of tossing a coin a large number of times systematically result in balanced histograms for heads and tails.

Logical [Degree of truth]

The degree of truth in the proposition "the coin will land heads" is the same as that of the analogous proposition regarding tails, before we have a chance to observe the outcome.

Subjective [A description of a person's beliefs]

I see no evidence to favor one outcome over another, and thus would be willing to take any side on a bet on the coin landing heads.

\subsubsection{Economists' mainstream interpretation}

Some phenomena may be contradictory to EU theory under a particular interpretation, while not under other interpretations. Consider, for instance, the purchase of lottery tickets. Suppose that the gambler does it for the money, not the fun of gambling. Under a classical interpretation, such behavior is paradoxical if we further assume pervasive risk aversion (a standard assumption), for such a transaction lowers the expected value and increases the risk of the individual's consumption bundle. Therefore, an EU-maximizer would never do it, under a classical interpretation. Under a subjective interpretation, on the other hand, there is no contradiction in assuming that all buyers believe they have higher chances of winning than other average buyers. The probability of winning is subjective and does not need to correspond to the ratio 1-over-the number of tickets sold. There is no conceptual problem in characterizing a situation in which all feel simultaneously luckier than the rest.

It should be stressed, however, that the economic profession generally welcomes the universality derived from Savage's development, but is reluctant to fully embrace the subjective view, mainly because there is the belief that taking subjectivism literally would render the theory almost empty: the "anything is possible" critique. In the previous example, the argument goes, if any belief is acceptable, then the theory has no empirical content. Ellsberg's experiment, however, falsifies this claim. 
In principle, there could be no connection between observed frequencies, however they may have been arranged, and beliefs. This is not to say that subjectivism holds that there is no link between beliefs and evidence, quite on the contrary. After all, Bayesianism obtained its name from Bayes' analysis of how rational people should interpret evidence and adjust beliefs accordingly. The point is that the learning procedure implied by Bayes' rule by itself does not restrict what an individual's beliefs should be at a certain point in time, even if we know what evidence he has been exposed to, without knowing his initial or prior beliefs, which need not be restricted by the data in any way.

Thus, popular applications of EU theory in all areas of economics impose either homogeneous beliefs - all individuals believe the same - or disparate information but common priors, so that everybody would believe the same if fed with the same information, the so-called Harsanyi doctrine. This is the case of rational expectation models in macroeconomics and finance (e.g., the CAPM, Grossman and Stiglitz, 1980 or Lucas, 1978), and game-theoretical models of information (e.g., Spence, 1973, Riley, 1979, and Vickrey, 1961). ${ }^{16}$

Furthermore, when these models are analyzed empirically, the individuals' beliefs are taken to correspond to suitably defined empirical frequencies. This is the case, for instance, with the Efficient Market Hypothesis discussion.

The following was an actual classroom discussion in a finance course. The professor asked "What is the variance of tomorrow's price of IBM shares?" A student replied that next day's IBM share price did not have a variance, that while possibly every person was uncertain about the value, the beliefs of each could be represented by a probability function, which in turn could have an associated variance. Not knowing the value of the variance of an individual's beliefs amounts to saying that his beliefs are unknown to the observer; if the individual of interest is oneself, then not knowing the variance means that one is uncertain about one's own beliefs - but at this point, the question of what that variance is loses its original appeal. Clearly, the student adhered to a subjectivist view, while his professor to an objective, propensity view. What is interesting about this anecdote is that it illustrates that, although in many cases a question asked under one view can easily be rephrased to make sense in another, there are some research questions that seem important under one view but empty under another. This is a consequence of the fact that economists sometimes use a common model for radically different theories.

The professor's understanding of probability seems pervasive among economists. Incidentally, when asked to choose which of the five interpretations in Table 14 best represents their understanding of the phrase "the probability of heads is $\frac{1}{2}$ ", the five colleagues I asked chose the second one, and four doubted 2 and 3 were equivalent. Of course this does not prove anything, but illustrates the idea that if there is a mainstream interpretation, it is of an objective nature.

\footnotetext{
${ }^{16}$ For a comprehensive discussion of the Common Prior Assumption in Economics, see Morris (1995). Gul (1998) and Aumann (1998) present opposite views on the role of subjectivism in economic theory.
} 
Apparently, then, economists welcomed the idea of applying EU pervasively to represent behavior under uncertainty, as it freed them from the narrow applicability sought by Keynes, for instance, but did not embrace the view about probability that supported such universality.

Thus, although the interpretation and applicability of probability to economics was the subject of much discussion in the first half of the $20^{\text {th }}$ century, by the second half the mathematical model had been accepted and was being widely applied (McGoun 1995). Since the debate over the meaning was never actually settled, the new mathematical models received ambiguous interpretations. Empiricists, on the other hand, embraced propensity views, even when the more natural interpretations of the models might be subjective, because of the need to have ready counterparts to the individual's beliefs in the data.

At any rate, the widespread adoption of probabilities to represent beliefs has the advantage of giving the modeler access to all the apparatus developed in mathematical probability theory and its application to statistics.

\subsection{Dynamic Decision}

There is yet another explanation. Most applications of EUT have to do with dynamic decisions, i.e., decisions in which the DM cannot commit to fulfilling a particular course of action but rather must sequentially confirm or revise any initial plans. Suppose the DM could choose a contingent plan of action at time 0 and was forced to fulfill it completely, until its end. Compare it with the sequence of decisions he would make if he could at different points in time change some parts of that plan. His decisions are said to be intertemporally, time, or dynamically consistent if in both cases he would implement exactly the same plan, that is, if the opportunity of revising his (contingent) decisions had no value for him.

Time consistency is of extraordinary importance to both, the predictive and the positive sides of any theory. From an empirical point of view, a time inconsistent decision rule should be much more difficult to estimate in general. More importantly, inconsistent choice opens the question as to whose incarnation of the DM should be considered when assessing how his welfare would be affected, say, by some policy measure or event under study. On the other hand, dynamically inconsistent behavior is generally thought to create "money pumps," whereby clever individuals could devise sequences of transaction proposals that would be accepted by the inconsistent $\mathrm{DM}$ and be profitable to the exploiting party; this constitutes a problem for models of economic interaction. ${ }^{17}$

The standard model posits a time additive expected utility function (possibly with discounting) like that of Equation 21.

\footnotetext{
${ }^{17}$ However, see Machina (1989) for a thorough discussion of this problem and the counterarguments. Also see Cubitt and Sugden (2001) for a critical assessment of general "money pump" arguments.
} 
(21) $\sum_{t=0}^{T} \delta^{t} E\left[u_{t} \mid h_{t}(\omega)\right]$

The utility function representing future preferences is obtained from this by updating the probabilities according to Bayes' rule. The choice of a utility function of the form of Equation 21, along with the updating of beliefs (and thereby of preferences) by Bayes' rule, is far from innocent: it is the only way of guaranteeing time-consistent decisions under expected utility maximization (Weller, 1978. See also Epstein and Le Breton, 1993). Sequences of beliefs that do not satisfy Bayes' rule do not necessarily generate time consistent decisions.

Of course it is an empirical issue whether observed decisions can be better described as time consistent or time inconsistent; but in view of these difficulties, the profession's stance appears to be to assume time consistency unless it becomes essential and untenable in the particular problem at hand.

From an objectivist's lens, probabilities exist and satisfy Bayes' rule (by Bayes' theorem). Hence, granted time additivity, von Neumann and Morgenstern's theory could be extended to characterize time consistent choice. From a subjectivist's point of view, probabilities do not exist per se, and it is an open question whether the implied beliefs of future decision moments would relate to the DM's past beliefs as predicted by Bayes' rule. Savage's theory, however, provides a strong foundation for assuming they are.

In truth, Savage's theory is static. Nevertheless, it can be interpreted as a dynamic one through the notion of conditional preferences, to be interpreted as the preference ranking over acts after learning that an event has occurred. The representation of this conditional preference also takes the form of an expected utility, obtained from the unconditional one by an update of the probability that satisfies the definition of conditional probability. Hence, the implied beliefs satisfy Bayes' rule, ${ }^{18}$ giving support to understand this as a dynamic theory in which inference from evidence is "reasonable."

Note also that if the information received by the DM is represented by a non partitional possibility correspondence, the DM's beliefs will fail to satisfy Bayes' rule. Hence both, the description of the evolution of knowledge as a sequence of partitions of increasing refinement discussed in Section 3, and the description of beliefs by sequences of probabilities over those partitions that

\footnotetext{
${ }^{18}$ This claim is in fact very imprecise (or simply false), as one referee pointed out. Savage's axioms imply the existence of a unique (up to positive linear transformations) expected utility representation of preferences where the felicity function is state independent. The satisfaction of Bayes' rule is granted for the associated probability. However, those axioms do not rule out the existence of other representations with state-dependent felicity functions; the probabilities associated to those representations do not satisfy Bayes' rule. It is conventional, but not required by the theory, to pick the state-independent representation; our claim is correct only within this convention. Only recently necessary and sufficient conditions for the existence, uniqueness, and updating, according to Bayes' rule, of subjective probabilities representing individual beliefs have been worked out (Karni, 2003).
} 
relate to each other according to Bayes' rule, are necessary for describing time consistent decisions within the EU framework.

It is unclear what the proper extension of Bayes' rule is to capacities. There are many proposals (Gilboa and Schmeidler, 1993; Fagin and Halpern, 1991; Epstein, 2002). In most instances, however, capacities do not generate time consistent decisions. In the multiple prior case, one could simply assume Bayesian updating prior by prior. Epstein and Schneider (2003) have axiomatized such dynamic representation of updating beliefs (and preferences). Moreover, they identified a set of sufficient conditions (on the set of prior beliefs) that guarantee time consistent decisions. The message is that some generalizations of EUT revive the issue of dynamic consistency, an issue that may be determinant to the adoption of particular generalizations.

\subsection{Applications of Ambiguity}

\subsubsection{The stock market participation puzzle}

The areas in which CEU theory yields interesting predictions are abundant. One example is offered by the stock market (non) participation puzzle. While EUTbased portfolio theory predicts that investors in perfectively competitive markets would hold every available asset in their portfolios (though in different proportions, accounting for differences in beliefs, risk aversion or endowments), typical households do not hold stocks. For instance, in the US only one of every five households does. ${ }^{19}$

In contrast, Dow and Werlang (1992) showed that if an investor had (convex) non-additive beliefs regarding assets' returns, then there would be a range of prices at which he would not buy or sell, but stay out of the market. Ambiguity aversion offers a rationalization of this puzzle. To see this, consider the following example.

\section{Example 3}

As in conventional portfolio theory, consider an investor with $\$ W_{0}$ available to invest in two assets, one of which is risky and the other riskless. Think of the former as a stock, and of the latter as a bond, with a short time horizon. Suppose there are two states, $\omega_{1}$ and $\omega_{2}$. Depending on the state, the gross payoff of the risky asset is either $\$ r_{1} \equiv r\left(\omega_{1}\right)$ or $\$ r_{2} \equiv r\left(\omega_{2}\right)$, with $r_{1}>r_{2}$; the riskless asset pays $\$ r$ regardless of the state. The riskless and the risky assets sell at $q_{0}$ and $q_{1}$ respectively at date 0 . The risk premium refers to the difference in expected rates of return: $\pi_{1} r_{1} / q_{1}+\pi_{2} r_{2} / q_{1}-r / q_{0}$.

\footnotetext{
${ }^{19}$ This figure corresponds to direct holdings, and is obtained from the Survey of Consumer Finances. See Naudon, Tapia and Zurita (2004).
} 
If $\pi_{l}$ is the probability of state $\omega_{l}$, then the expected utility of investing a fraction $\alpha$ in the risky asset and the remaining $(1-a)$ in the riskless asset is given by:

$$
\pi_{1} u\left(W_{0}\left((1-\alpha) \frac{r}{q_{0}}+\alpha \frac{r_{I}}{q_{1}}\right)\right)+\left(1-\pi_{1}\right) u\left(W_{0}\left((1-\alpha) \frac{r}{q_{0}}+\alpha \frac{r_{2}}{q_{1}}\right)\right)
$$

Starting from a riskless portfolio, the investor would like to spend some money on the risky asset if $\left.\frac{\partial E[u]}{\partial \alpha}\right|_{\alpha=0}>0$, which amounts to:

$$
\pi_{1} u^{\prime}\left(W_{0}\left(\frac{r}{q_{0}}\right)\right) W_{0}\left(\frac{r_{1}}{q_{l}}-\frac{r}{q_{0}}\right)+\left(1-\pi_{l}\right) u^{\prime}\left(W_{0}\left(\frac{r}{q_{0}}\right)\right) W_{0}\left(\frac{r_{2}}{q_{1}}-\frac{r}{q_{0}}\right)>0
$$

Rearranging, we get:

$$
\pi_{l}\left(\frac{r_{1}}{q_{1}}\right)+\pi_{2}\left(\frac{r_{2}}{q_{1}}\right)-\frac{r}{q_{0}}>0
$$

This is to say, an investor would spend some of his money on the risky asset if this offers a positive risk premium. Observe that this result does not depend on the degree of risk aversion. Hence, if there is a risk premium, the investor would always hold some of the risky asset in his portfolio, i.e., he would participate on the stock market.

Consider instead a DM whose beliefs are represented by a capacity like the one in Table 15.

TABLE 15

A CAPACITY

\begin{tabular}{c|c}
$E$ & $v(E)$ \\
\hline$\varnothing$ & 0 \\
$\left\{\omega_{l}\right\}$ & $v_{l}$ \\
$\left\{\omega_{2}\right\}$ & $v_{2}$ \\
$\left\{\omega_{l}, \omega_{2}\right\}$ & 1
\end{tabular}

If the capacity is convex (i.e., if $v_{l}+v_{2}<1$ ), then the CEU would put all unassigned weight to the worst case scenario, creating a kink in the indifference curve at the certainty line: 


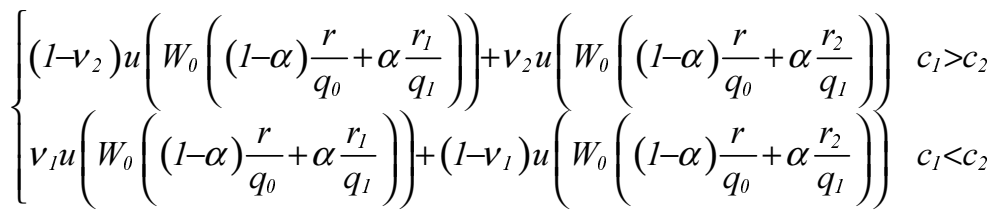

Hence, the utility function is non-differentiable on that line. The directional derivative from the right $(\alpha>0)$ yields in this case:

$$
\left(1-v_{2}\right) u^{\prime}\left(W_{0}\left(\frac{r}{q_{0}}\right)\right) W_{0}\left(\frac{r_{1}}{q_{1}}-\frac{r}{q_{0}}\right)+v_{2} u^{\prime}\left(W_{0}\left(\frac{r}{q_{0}}\right)\right) W_{0}\left(\frac{r_{2}}{q_{1}}-\frac{r}{q_{0}}\right)>0
$$

That is, the individual would buy the risky asset as long as:

$$
\left(1-v_{2}\right)\left(\frac{r_{1}}{q_{l}}\right)+v_{2}\left(\frac{r_{2}}{q_{1}}\right)-\frac{r}{q_{0}}>\begin{array}{c|ccc}
c & c & d \\
d & 9,9 & 0,8 \\
8,0 & 7,7
\end{array}
$$

A similar analysis reveals that he would (short) sell it if:

$$
v_{l}\left(\frac{r_{1}}{q_{1}}\right)+\left(1-v_{l}\right)\left(\frac{r_{2}}{q_{1}}\right)-\frac{r}{q_{0}}<0
$$

Hence, there is a range of prices where the investor would not participate in the risky asset market, defined by the inequality:

$$
\left(1-v_{2}\right)\left(\frac{r_{1}}{q_{1}}\right)+v_{2}\left(\frac{r_{2}}{q_{1}}\right)>\frac{r}{q_{0}}>v_{l}\left(\frac{r_{1}}{q_{1}}\right)+\left(1-v_{1}\right)\left(\frac{r_{2}}{q_{1}}\right)
$$

This means that if the risk premium is positive but not high enough, the DM does not participate. Observe that the range would be null if $\left(1-v_{2}\right)=v_{1}$, that is, if the capacity were additive (i.e., a probability), as asserted before. Observe also that as the ambiguity increases so does the range of non-participation prices. Naudon et al. (2004) observe that the strong empirical connection between education and participation could be rationalized if the degree of ambiguity perceived in financial risks were related to the education of the DM.

It is easy to see also that the same would happen if the DM had a set of priors instead of a capacity, as in the urn example. 


\subsubsection{Ambiguous games}

Marinacci (2000) analyzes games where beliefs are ambiguous. Consider the stag hunt game:

TABLE 16

PAYOFF MATRIX FOR THE STAG HUNT GAME

\begin{tabular}{c|cc} 
& $c$ & $d$ \\
\hline$c$ & 9,9 & 0,8 \\
$d$ & 8,0 & 7,7
\end{tabular}

This is a classical coordination game: each player would like to do exactly what his opponent does, but in principle has no clue as to how to predict her behavior. It has two (pure strategy) Nash equilibria: $(c, c)$ and $(d, d)$. The $(c, c)$ equilibrium leads to the best possible payoff (indeed, it is the only efficient outcome), but is risky in the sense that if one player is mistaken about her opponent's play, she gets 0 . Action $d$, on the other hand, gives her at least a payoff of 7; and in the Nash equilibrium $(d, d)$ being mistaken about the opponent's behavior would lead to a better payoff. $(d, d)$ is in fact the risk-dominant equilibrium. ${ }^{20}$

Ambiguity aversion may actually help select among Nash equilibria in games of this sort. Recall that either CEU or MEU result in a maxmin decision rule in the limit as ambiguity becomes large and the DM's attitude towards it is pessimistic. In that case, the CEU or the MEU associated to action $c$ would be 0 while for action $d 7$, hence the only equilibrium would be the risk-dominant one. On the other hand, under extreme optimism the $(c, c)$ equilibrium would be selected.

In a similar fashion, Eichberger et al. (2005) revisit classic games like that of oligopoly in the views of Cournot and Bertrand. Depending on the players' attitudes towards ambiguity, equilibrium prices might be higher or smaller that under EU maximization.

The message to grasp is that, when ambiguity is considered, the strategic form does not contain all relevant information to predict the outcome of the game, for it does not include the description of the ambiguity perceived by the players and their attitudes towards it.

\subsubsection{General equilibrium under ambiguity}

The consequences of ambiguity have been explored in general equilibrium theory as well. For instance, Epstein (2001) develops a highly stylized model where the low empirical correlation of consumption across countries can be

\footnotetext{
${ }^{20} \mathrm{~A}$ Nash equilibrium is said to dominate in risk a second Nash equilibrium if the probability of being mistaken needed to convince the player to switch to another strategy is higher. In this case, abandoning the $\mathrm{c}$ strategy is optimal if the probability that the opponent plays $\mathrm{d}$ necessary to convince the player to change her decision is $1 / 5$, while for $\mathrm{d}$ to switch to $\mathrm{c}$ is $7 / 8$.
} 
explained. This empirical regularity, sometimes referred to as the home-bias puzzle, ${ }^{21}$ is a long-standing puzzle in international economics. It is puzzling under the lens of general equilibrium theory with EU maximizers, where risk-sharing opportunities are obvious gains from trade: countries should insure each other against idiosyncratic shocks.

Mukerji and Tallon (2001) show an interesting feature of diversification under ambiguity. They point out that the Law of Large Numbers in this setting says that the mean of a sequence of independent and identically distributed random variables converges to their expected value just as with normal probabilities, except for the fact that being now the set of probabilities non singleton, the convergence to the mean is actually to a set of means. This can be though of as saying that while the investors in such economy know that trough diversification the risk in their portfolios may become arbitrarily small, they don't know to which mean the returns converge to; a MEU would evaluate those portfolios according to their lowest possible mean. Ambiguity aversion then has bite in explaining investor's behavior even in economies where almost perfect diversification is possible.

\section{FinAL REMARKS}

The development of the EU-model and its associated theories is possibly one of the greatest intellectual achievements of the 20th century. During the second half, it made possible the development of entire fields, among them financial economics, game theory, the economics of information, and modern macroeconomics. Some questions still remain, however, that it has not proved capable of answering in its present form, particularly in situations in which the uncertainty goes "beyond earthquakes and the weather."

This essay discussed separately two aspects of individual decision making with potentially important behavioral content: unawareness and ambiguity. It turns out that these may be intimately connected, as Ghirardato's (2001) research suggests.

Ghirardato adapted Savage's model to the unawareness case. His construction consists of separating the individual's subjective state space from the objective state space. The former reflects the DM's understanding of the problem, while the latter the true possibilities. Given that the DM's understanding is limited, his state space is coarser than the objective space. Thus, the consequence of an act $c(a, \omega)$ is unknown even when conditional on a state; mathematically, $c(a, \omega)$ is a set instead of a point. After extending Savage's axioms he obtains the same EU representation of preferences and therefore a probabilistic representation of beliefs, but over the subjective space. Adding some more structure he manages to obtain another representation, this time over the objective state space, which

\footnotetext{
${ }^{21}$ There is a related puzzle in finance under that name: the fact that investors typically concentrate their portfolios in domestic assets much more than the benefits of diversification would recommend.
} 
turns out to be non-additive. Hence, an observer might be able to represent a subject's behavior by means of a CEU because of the subject's unawareness. ${ }^{22}$

At any rate, these extensions of EUT are an active research field, which is likely to produce new answers to old problems. Similarly, the associated development of new tools will make economic theory suitable for the analysis of new problems as well, expanding its frontiers, just as EUT itself did in the past.

\section{REFERENCES}

Ahn, D. (2005), “Ambiguity without a State Space," mimeo. University of California, Berkeley.

Allen, F. and D. Gale (1989), "Optimal Security Design,” The Review of Financial Studies, 1: $229-263$.

Aumann, R. (1998), “Common Priors: A Reply to Gul," Econometrica, 66: 929-938.

Aumann, R. (1999a), "Interactive Epistemology I: Knowledge," International Journal of Game Theory, 28: 263-300.

Aumann, R. (1999a), "Interactive Epistemology II: Probability," International Journal of Game Theory, 28: 301-314.

Arrow, K., and F. Hahn (1999), "Notes on Sequence Economies, Transaction Costs and Uncertainty," Journal of Economic Theory, 86: 203-218.

Bernardo, J. and A. Smith (1994), Bayesian Theory, J. Wiley \& Sons.

Bernoulli, D. (1738), "Exposition of a New Theory on the Measurement of Risk," reprinted and translated in Econometrica, 22: 23-36, 1954.

Camerer, C. (1995), "Individual Decision Making," in The Handbook of Experimental Economics, edited by J. Hagel and A. Roth, Princeton University Press.

Casadesus-Masanell, R., P. Klibanoff and E. Ozdenoren (2000), "Maxmin Expected Utility over Savage Acts with a Set of Priors," Journal of Economic Theory, 92: 35-65.

Cubitt, R. and R. Sugden (2001), "On Money Pumps," Games and Economic Behavior, 37: 121-160.

Dekel, E., B. Lipman, and A. Rustichini (1998a), "Standard State-Space Models Preclude Unawareness," Econometrica, 66: 159-173.

Dekel, E., B. Lipman, and A. Rustichini (1998b), "Recent Developments in Modeling Unforeseen Contingencies," European Economic Review, 42: 523-542.

Dow, J. and Werlang, S.R. (1992), "Uncertainty Aversion, Risk Aversion, and the Optimal Choice of Portfolio," Econometrica, 60: 197-204.

Eichberger, J., D. Kelsey and B. Schipper (2005), "Ambiguity and Social Interaction," mimeo.

Ellsberg, D. (1961), "Risk, Ambiguity, and the Savage Axioms," Quarterly Journal of Economics 75: 643-669.

Ely, J. (1998), “A Note on Unawareness," mimeo, Northwestern University.

Epstein, L. (2001), "Sharing Ambiguity," American Economic Review, 91: 45-50.

${ }^{22}$ It should be stressed, however, that his notion of unawareness is not epistemic as HMS's and is therefore subject to the DLR critique. 
Epstein, L. (2002), “An Axiomatic Model of Non-Bayesian Updating," Working Paper 498, University of Rochester.

Epstein, L. and M. Le Breton (1993), "Dynamically Consistent Beliefs must be Bayesian," Journal of Economic Theory, 61: 1-22.

Epstein, L. and M. Schneider (2003), "Recursive Multiple Priors," Journal of Economic Theory, 113: 1-31.

Fagin, R. and J. Halpern (1991), “A New Approach to Updating Beliefs," in P.P. Bonissone, M. Henrion, L.N. Kanal, and J. Lemmer, editors, Uncertainty in Artificial Intelligence, Volume VI, 347-374. Elsevier Science Publishers, Amsterdam.

Friedman, M. (1953), "The Methodology of Positive Economics," in Essays in Positive Economics, Chicago.

Fudenberg, D. and D. Levine (1993), "Self-Confirming Equilibrium," Econometrica, 61: $523-545$.

Fudenberg, D. and D. Levine (1995), "Consistency and Cautious Fictitious Play," Journal of Economic Dynamics and Control, 19: 1065-1090.

Fudenberg, D. and D. Levine (2005), "Learning and Belief Based Trade", Cuadernos de Economía-Latin American Journal of Economics, this issue.

Geanakoplos, J. (1989), "Game Theory without Partitions, and Applications to Speculation and Consensus," Cowles Foundation Discussion Paper No. 914.

Ghirardato, P. (1993), "Non-Additive Measures of Uncertainty: A Survey of Some Recent Developments in Decision Theory," International Review of Economics and Business, 40: 253-276.

Ghirardato, P. (2001), "Coping with Ignorance: Unforeseen Contingencies and Non-additive Uncertainty," Economic Theory, 17: 247-276.

Gilboa, I. and D. Schmeidler (1989), "Maxmin Expected Utility with a Non-Unique Prior," Journal of Mathematical Economics, 18: 141-153.

Gilboa, I. and D. Schmeidler (1993), "Updating Ambiguous Beliefs," Journal of Economic Theory, 59: 33-49.

Grossman, S. and J. Stiglitz (1980), "On the Impossibility of Informationally Efficient Markets," American Economic Review, 70: 393-408.

Gul, F. (1992), "Savage's Theorem with a Finite Number of States," Journal of Economic Theory, 57: 99-110.

Gul, F. (1998), “A Comment on Aumann's Bayesian View," Econometrica, 66: 923-927.

Hart, O. and J. Moore (1988), "Incomplete Contracts and Renegotiation," Econometrica, 56: 755-785.

Hájek, A. (2002), "Interpretations of Probability," Stanford Encyclopedia of Philosophy, E. Zalton (ed.).

Heifetz, Meier, M., and B. Schipper (2005), "Interactive Unawareness," Journal of Economic Theory, forthcoming.

Hirshleifer, J. (1977), "The Theory of Speculation under Alternative Regimes of Markets," Journal of Finance, 32: 975-999.

Karni, E. (2003), "Foundations of Bayesian Theory," forthcoming Journal of Economic Theory.

Knight, F. (1921), "Risk, Uncertainty and Profit.” Cosimo Classics (2002).

Lintner, J. (1969), “The Aggregation of Investors' Diverse Judgments and Preferences in Purely Competitive Security Markets," Journal of Financial and Quantitative Analysis, 4: 103-124.

Lucas, R. (1978), “Asset Prices in an Exchange Economy”, Econometrica, 46: 1429-1445.

Machina, M. (1989), "Dynamic Consistency and Non-Expected Utility Models of Choice under Uncertainty," Journal of Economic Literature, 27: 1622-1668. 
Marinacci, M. (2000), "Ambiguous Games," Games and Economic Behavior, 31: 191-219. Maskin, E. and J. Tirole (1999), "Unforeseen Contingencies and Incomplete Contracts," Review of Economic Studies, 66: 83-114.

McGoun, E. (1995), "The History of Risk Measurement," Critical Perspectives on Accounting, 6: 511-532.

Milgrom, P. and N. Stokey (1982), "Information, Trade, and Common Knowledge," Journal of Economic Theory, 26: 17-27.

Morris, S. (1995), "The Common Prior Assumption in Economic Theory," Economics and Philosophy, 11:227-253.

Mukerji, S. and J.M. Tallon (2001), "Ambiguity Aversion and Incompleteness of Financial Markets," The Review of Economic Studies, 68: 883-904.

Nakamura, Y. (1990), "Subjective Expected Utility with Non.additive Probabilities on Finite State Spaces," Journal of Economic Theory, 51: 346-366.

Naudon, A., M. Tapia and F. Zurita (2004), "Ignorance, Fixed Costs, and the Stock Market Participation Puzzle," PUC-Economics Working Paper 262.

Osborne, M. and A. Rubinstein (1994), "A Course in Game Theory," MIT.

Radner, R. (1979), "Rational Expectations Equilibrium: Generic Existence and the Information Revealed by Prices," Econometrica, 47: 655-678.

Riley, J. (1979), "Informational Equilibria," Econometrica, 47: 331-360.

Savage, L. (1957), "The Foundations of Statistics," Wiley. Revised edition, Dover, 1972.

Spence, M. (1973), “Job Market Signalling," Quarterly Journal of Economics, 87: 296-332.

Schmeidler, D. (1989), "Subjective Probability and Expected Utility without Additivity," Econometrica, 57: 571-587.

Vickrey, W. (1961), "Counterspeculation, Auctions, and Competitive Sealed Tenders," Journal of Finance, 16: 8-37

Von Neumann, J. and O. Morgenstern (1946), The Theory of Games, Princeton University Press.

Weller, P. (1978), "Consistent Intertemporal Decision-Making under Uncertainty," The Review of Economic Studies, 45: 263-266. 